J. DIFFERENTIAL GEOMETRY

55 (2000) 193-227

\title{
MODULI OF SHEAVES ON SURFACES AND ACTION OF THE OSCILLATOR ALGEBRA
}

\author{
VLADIMIR BARANOVSKY
}

\begin{abstract}
This paper gives a generalization of some results on Hilbert schemes of points on surfaces. Let $M^{G}(r, n)$ (resp. $M^{U}(r, n)$ ) be the Gieseker (resp. Uhlenbeck) compactification of the moduli spaces of stable bundles on a smooth projective surface. We show that, for surfaces satisfying some technical condition:
\end{abstract}

(a) The natural map $M^{G}(r, n) \rightarrow M^{U}(r, n)$ generalizing the HilbertChow morphism from the Hilbert scheme of $\mathrm{n}$ points on $\mathrm{S}$ to the n-th symmetric power, is strictly semi-small in the sense of GoreskyMacPherson with respect to some stratification.

(b) Let $P_{t}(X)$ be the Intersection Homology Poincare polynomial of $X$. Generalizing the computation due to Gottsche and Sorgel we prove that the ratio $\frac{\sum_{n} q^{n} P_{t}\left(M^{G}(r, n)\right)}{\sum_{n} q^{n} P_{t}\left(M^{U}(r, n)\right)}$ is a character of a certain Heisenberg-type algebra.

(c) Generalizing results of Nakajima we show how to obtain the action of the Heisenberg algebra on the cohomology using correspondences.

\section{Introduction}

Let $S$ be a smooth complex projective surface and $\operatorname{Hilb}^{n}(S)$ the Hilbert scheme of length $n$ zero-dimension subschemes of $S$. It is known (cf. [10]) that $\operatorname{Hilb}^{n}(S)$ is a smooth projective variety of dimension $2 n$. The structure of the cohomology $\operatorname{ring}$ of $\operatorname{Hilb}^{n}(S)$ for a fixed $n$ is rather difficult to understand. However, when we consider the direct sum $\bigoplus_{n \geq 0} H^{*}\left(\operatorname{Hilb}^{n}(S)\right)$ of the cohomology groups (always with complex

Received May 23, 1999. 
coefficients in this paper) over all possible $n$, the picture becomes more comprehensible.

For any complex smooth algebraic variety $X$ of dimension $d$ denote by $P_{t}(X)$ the shifted Poincaré polynomial $\sum_{i=0}^{d} t^{i-d} \cdot \operatorname{dim}_{\mathbb{C}} H^{i}(X)$. It was shown by Göttsche [Gö1] that, for any smooth quasi-projective surface $S$ one has an identity

$$
\sum_{n \geq 0} q^{n} P_{t}\left(\operatorname{Hilb}^{n}(S)\right)=\prod_{l=1}^{\infty} \frac{\left(1+t^{-1} q^{l}\right)^{b_{1}(S)}\left(1+t q^{l}\right)^{b_{3}(S)}}{\left(1-t^{-2} q^{l}\right)^{b_{0}(S)}\left(1-q^{l}\right)^{b_{2}(S)}\left(1-t^{2} q^{l}\right)^{b_{4}(S)}}
$$

where $b_{i}(S)$ are the Betti numbers of $S$.

Vafa and Witten [32] have noticed that the right hand side of the formula above is an irreducible character of the oscillator algebra (or Heisenberg/Clifford algebra) $\mathcal{H}$ defined for a smooth projective surface $S$ as follows:

(a) $\mathcal{H}$ is generated by elements $p_{i}^{\alpha}, \alpha \in H^{*}(S), i \in \mathbb{Z} \backslash 0$.

(b) The generators satisfy $\left[p_{i}^{\alpha}, p_{j}^{\beta}\right]=\langle\alpha, \beta\rangle i \cdot \delta_{i+j, 0}$,

where the commutator is understood in the graded sense and $\langle\cdot, \cdot\rangle$ is the intersection form on $S$.

One could expect to construct an actual action of the oscillator algebra on the cohomology groups, since one of the standard realizations of the Fock representation is in the space of all symmetric polynomials in infinitely many variables. This realization appears from the cohomology of symmetric powers $\operatorname{Sym}^{n}(S)$ related to Hilbert schemes of points via the Hilbert-Chow morphism $\operatorname{Hilb}^{n}(S) \rightarrow \operatorname{Sym}^{n}(S)$.

And indeed, Nakajima and Grojnowski (cf. [27], [15]) have constructed the expected action of $\mathcal{H}$ on $\bigoplus_{n \geq 0} H^{*}\left(\operatorname{Hilb}^{n}(S)\right)$ using some explicit cycles in the products of Hilbert schemes.

The Hilbert scheme can be viewed as a moduli space of rank one sheaves on $S$ with trivial determinant and $c_{2}=n$. It was conjectured by Vafa and Witten in [32] (see also [27], [15]) that there should be a higher rank extension of the results above. This paper provides such a generalization (cf. Theorem 4.1). The key obsevation which stands behind our arguments is that in certain cases a non-compact moduli space $\mathcal{M}$ may admit two different natural compactifications $\mathcal{M}_{1}, \mathcal{M}_{2}$ 
and a map $\mathcal{M}_{1} \rightarrow \mathcal{M}_{2}$ which (partially) resolves the singularities of $\mathcal{M}_{2}$ and restricts to identity on the copies of $\mathcal{M}$ in $\mathcal{M}_{1}$ and $\mathcal{M}_{2}$.

The list of examples includes moduli of maps of curves to flag varieties $\left(\mathcal{M}_{1}\right.$ and $\mathcal{M}_{2}$ are Laumon and Drinfeld compactifications, respectively, cf [20]), instantons on ALE spaces (quiver variety and the Uhlenbeck-type compactification cf. [26]), moduli of abelian varieties (Voronoi and Satake compactifications) and, finally, moduli of stable bundles on surfaces (Gieseker and Uhlenbeck compactifications). Thus, the Hilbert scheme is replaced by the Gieseker moduli space $M^{G}(r, n)$ of stable torsion-free sheaves and the role of the symmetric power is played by the Uhlenbeck compactification $M^{U}(r, n)$.

The first key result of this paper is that the fibers of the natural map $M^{G}(r, n) \rightarrow M^{U}(r, n)$ are irreducible of expected dimension. (This generalization of the results by Briançon and Iarrobino (cf. [5], [18]) was also proved independently by Ellingsrud and Lehn, cf. [9]).

This leads to the second important theorem which says that, in the coprime case, the natural morphism $M^{G}(r, n) \rightarrow M^{U}(r, n)$ is strictly semi-small in the sense of Goresky-MacPherson (if a certain technical condition is satisfied: the surface $S$ is allowed, for instance, to be rational, birationally ruled, K3 or abelian). This result was originally conjectured by V. Ginzburg and the corresponding statement is true for some of the moduli spaces mentioned above. We proceed further to give a natural generalization of the rank one correspondences yielding an action of the oscillator algebra on $\bigoplus_{n \geq 0} H^{*}\left(M^{G}(r, n)\right)$. We show how to extend Nakajima's proof of commutation relations to the higher ranks case. Some parts of the proof require a more detailed analysis of the geometry of moduli spaces, than in the case of Hilbert schemes (see Sections 5 and 6).

It was communicated to us by $\mathrm{G}$. Moore that $L^{2}$-cohomology groups of instanton moduli spaces have recently appeared in string theory. Since $L^{2}$-cohomology of a non-compact moduli space coincides in several interesting cases with intersection homology of its particular compactification, this provides another motivation for studying these homology groups. This work can be regarded as a first step in application of intersection homology to moduli spaces of sheaves on surfaces.

Acknowledgements. I would like to thank Victor Ginzburg whose conjecture has originally motivated this work. I am grateful to Lothar Göttsche, Stein Arild Strømme, Zhenbo Qin, Gregory Moore 
and Hiraku Nakajima for the useful discussions, advices and remarks.

A part of this work was carried out during my stay at the Max Planck Institut für Mathematik in Bonn. I want to thank all the people involved, particularly Don Zagier, for the invitation and the excellent research conditions.

\section{The moduli spaces}

Let $S$ be a smooth complex projective surface. We are interested in the study of the moduli space of rank $r$ vector bundles on $S$. It is known, cf. [22], that all bundles cannot be parametrized by points of an algebraic variety, because of the problems arising from vector bundles with large automorphism groups. Instead one restricts attention only to "good" bundles, i.e those which are Gieseker-stable (cf. [17]) with respect to a fixed ample line bundle $H$. Moreover, in this paper we will only consider the situation when all other notions of "good" bundles, Gieseker semistability and Mumford (semi)stability, coincide with Gieseker stability (see next section).

Thus, we consider the moduli space $N(r, n)$ of Gieseker $H$-stable vector bundles $E$ of rank $r$ with fixed determinant $L$ and $c_{2}(E)=n$. The line bundles $L$ and $H$ will be fixed throughout this paper. It is known, (cf. for example [17]), that $N(r, n)$ is empty unless the following Bogomolov-Miyaoka inequality is satisfied

$$
n-\frac{r-1}{2 r} c_{1}(L)^{2} \geq 0
$$

The moduli space $N(r, n)$ is non-compact and it can be compactified in two different ways. The first compactification is the Gieseker moduli space $M^{G}(r, n)$ of all $H$-stable torsion-free sheaves $E$ of rank $r$ with $\operatorname{det}(E)=L$ and $c_{2}(E)=n$ (cf. [22]).

The second compactification is the Uhlenbeck moduli space $M^{U}(r, n)$ which can be described as follows. Denote by $\operatorname{Sym}^{k} S$ the $k$-the symmetric power of $S$, and start with the disjoint union $\coprod_{s=0}^{\infty} N(r, n-s) \times$ $\mathrm{Sym}^{s} S$ (note that this union is in fact finite by (1)). A theorem due to Uhlenbeck [31] says that any sequence of points in one piece of this disjoint union has a subsequence that converges (in some sense) to a

point in another piece. This endows $\bigcup_{s=0}^{\infty} N(r, n-s) \times \operatorname{Sym}^{s} S$ with a topology of a compact space. One can show (cf. [17, Chapter 8]) that this topological space can be endowed with a structure of an algebraic 
variety. This variety is called the Uhlenbeck compactification $M^{U}(r, n)$ of $N(r, n)$.

In general, the two compactifications above may be quite difficult to investigate: $N(r, n)$ may not be dense in $M^{G}(r, n)$ or $M^{U}(r, n)$, there may be components of dimension higher than expected, etc. Hence we impose a technical condition which ensures that the moduli spaces are well-behaved.

\section{The Main Technical Condition}

(a) The integers $r$ and $d:=c_{1}(L) \cdot c_{1}(H)$ are coprime.

(b) Either the canonical bundle $K_{S}$ is trivial or $c_{1}\left(K_{S}\right) \cdot c_{1}(H)<0$.

The main reason for imposing (a) and (b) is that they guarantee (cf. [17]) that for any $n$ the moduli space $M^{G}(r, n)$ is either empty or smooth of expected dimension

(2) $\operatorname{dim} M^{G}(r, n)=2 r n-(r-1)\left(c_{1}(L)\right)^{2}-\left(r^{2}-1\right) \chi\left(\mathcal{O}_{S}\right)+h^{1}\left(\mathcal{O}_{S}\right)$.

Condition (b) is automatic when $(-K)$ can be represented by an effective curve. Moreover, since $N(r, n)$ is an open subset of $M^{G}(r, n)$ (loc. cit.), it is also smooth.

Since $r$ and $d$ are assumed to be coprime, the two possible notions of stability (Mumford-Takemoto and Gieseker) coincide.

Examples. If we require that $K_{S}$ is trivial or effective then $S$ can be a Del Pezzo surface, K3 or abelian surface. If we make a special choice of $H$ as above, the list of examples will extend to all rational surfaces and birationally ruled surfaces.

It is known (e.g., $[8,10.3 .4])$ that for some elliptic surfaces or surfaces of general type the condition (2) may fail.

\section{Gieseker to Uhlenbeck}

By definition the Hilbert scheme $\operatorname{Hilb}^{n}(S)$ parametrizes Artin subschemes $\xi \in S$ of length $n$. Every such subscheme is determined by its ideal sheaf $J_{\xi} \subset \mathcal{O}_{S}$. The sheaves $J_{\xi}$ which arise in this way can be characterized as torsion-free sheaves of rank one with trivial determinant and $c_{2}=n$.

Thus, $M^{G}(r, n)$ is a higher rank analogue of $\operatorname{Hilb}^{n}(S)$. We want to generalize the natural map $a_{1}: \operatorname{Hilb}^{n}(S) \rightarrow \operatorname{Sym}^{n}(S)$ given by

$$
a_{1}: J_{\xi} \mapsto \sum_{x_{i} \in \text { Supp } \xi} \text { mult }_{x_{i}}(\xi) \cdot x_{i}
$$


where the formal sum on the left hand side is viewed as an element of $\operatorname{Sym}^{n}(S)$ (the rigorous construction of $a_{1}$ uses the notion of "linear determinant", see [17]).

To define the generalization $a_{r}: M^{G}(r, n) \rightarrow M^{U}(r, n)$ of the map $a_{1}$, for every torsion-free sheaf $\mathcal{F} \in M^{G}(r, n)$ we consider the double dual $\mathcal{F}^{* *}$. Since $\mathcal{F}^{* *}$ is reflexive (i.e., coincides with its own double dual) and $\operatorname{dim} S=2$, we can apply Lemma 1.1.10 in [28] and conclude that $\mathcal{F}^{* *}$ is locally free.

Now consider the short exact sequence:

$$
0 \rightarrow \mathcal{F} \rightarrow \mathcal{F}^{* *} \rightarrow A_{\mathcal{F}} \rightarrow 0
$$

The quotient sheaf $A_{\mathcal{F}}=\mathcal{F}^{* *} / \mathcal{F}$ is an Artin sheaf of a certain length $l_{\mathcal{F}}$ supported by finitely many points with multiplicities which add up to $l_{\mathcal{F}}$. We define $a_{r}(\mathcal{F}) \in M^{U}(r, n)$ by

$$
a_{r}: \mathcal{F} \mapsto\left(\mathcal{F}^{* *}, \sum_{x_{i} \in \text { Supp } A_{\mathcal{F}}} \operatorname{mult}_{x_{i}}\left(A_{F}\right) \cdot x_{i}\right)
$$

where the image is a point in $N\left(r, n-l_{\mathcal{F}}\right) \times \operatorname{Sym}^{l_{\mathcal{F}}}(S) \subset M^{U}(r, n)$.

Remark. Another consequence of $r$ and $d=c_{1}(H) \cdot c_{1}(L)$ being coprime is that any semistable sheaf is necessarily stable. This ensures that $\mathcal{F}^{* *}$ is stable if $\mathcal{F}$ is, hence the definition above makes sense. If $r$ and $d$ are not coprime, the space $M^{G}(r, n)$ parametrizes only S-equivalence classes (cf. [22]) of semistable sheaves and one has to be more careful to make sure that $a_{r}$ is well-defined on such classes (cf. [21]).

The algebraic structure on $M^{U}(r, n)$ is defined in such a way that the map $a_{r}$ is automatically algebraic (cf. [17, Chapter 8] and also the orginal paper by J. Li [21]).

Now we describe the fiber of $a_{r}$ over an arbitrary point $p=$ $\left(E, \sum_{i} m_{i} x_{i}\right)$ in $M^{U}(r, n)$. Here $m_{i}$ are positive integers and the points $x_{i}$ are pairwise distinct. The fiber $a_{r}^{-1}(p)$ parametrizes all the quotients $E \rightarrow A \rightarrow 0$ where the sheaf $A$ is supported at the points $x_{i}$ with prescribed multiplicities $m_{i}$. Since the question is local, the fiber does not depend on $E$ and points $x_{i}$ but only on $r$ and $m_{i}$. To be more precise, let Quot $(r, n)$ denote the punctual Quot scheme of all quotients $\mathcal{O}^{\oplus r} \rightarrow A$ of fixed length $n$ which are supported at a fixed point $x$. This scheme depends only on completion $\widehat{\mathcal{O}}_{x}$ of the local ring $\mathcal{O}_{x}$ of $x$, hence different points $x$ lead to isomorphic Quot schemes. Since every vector bundle is locally trivial by definition, the following statement is immediate 
Proposition 2.1. The fiber $a_{r}^{-1}(p)$ over the point $p=\left(E, \sum_{i} m_{i} x_{i}\right)$ is isomorphic to the product of punctual Quot schemes $\prod_{i}$ Quot $\left(r, m_{i}\right)$.

The proposition above motivates the following theorem (cf. [2] and $[9])$ :

Theorem 2.2. The punctual Quot scheme Quot $(r, n)$ defined above, is irreducible of dimension $r n-1$.

Proof. See Appendix. q.e.d.

The first application of Theorem 2.2 is to show that, under the Main Technical Condition, the moduli spaces $M^{G}(r, n)$ and $M^{U}(r, n)$ are well-behaved.

Theorem 2.3. Assume that the Main Technical Condition of Section 1 is satisfied. Then the following statements hold:

(a) $N(r, n)$ is dense in $M^{G}(r, n)$;

(b) $N(r, n)$ is dense in $M^{U}(r, n)$;

(c) Any irreducible component of $N(r, n-s) \times \operatorname{Sym}^{s}(S)$ intersects the closure of a unique component of $N(r, n)$ (and hence by $(\mathrm{b})$ is contained in it).

Proof. Note that (a) implies (b) since $a_{r}$ is surjective and one-to-one on the copies of $N(r, n)$ in $M^{G}(r, n)$ and $M^{U}(r, n)$, respectively.

To prove (a) suppose that $N(r, n)$ is not dense in $M^{G}(r, n)$. Then there exists a component of $M^{G}(r, n)$ such that generic point of it corresponds to a non-locally free sheaf. This means that the image of this component in $M^{U}(r, n)$ is a subset of $\bigcup_{i \geq 1} N(r, n-s) \times \operatorname{Sym}^{s}(S)$. Since all the components of $N(r, n)$ have expected dimension (and not more than that), Theorem 2.2 above implies that the dimension of $a_{r}^{-1}\left(\bigcup_{i \geq 1} N(r, n-s) \times \operatorname{Sym}^{s}(S)\right)$ is strictly less than the expected dimension of $M^{G}(r, n)$ which is impossible.

Finally, if (c) were false, some irreducible component $X$ of $N(r, n-$ $s) \times \operatorname{Sym}^{s}(S)$ would intersect the closures of at least two different components of $N(r, n)$. Since $M^{G}(r, n)$ is smooth, these would mean that $X$ would belong to the image of two different connected components of $M^{G}(r, n)$. But this is impossible since all fibers of $a_{r}$ are irreducible.

q.e.d.

Corollary 2.4. $M^{U}(r, n)$ is a disjoint union of the closures of irreducible components of $N(r, n)$. 


\section{Stratifications and semi-small maps}

From now on we will assume that the Main Technical Condition on the pair $(S, H)$ is satisfied.

Recall briefly the results on symmetric products and Hilbert schemes.

Let $\mu$ be a partition of $n$. Any such $\mu$ can be represented either by a non-increasing sequence $\mu=\left(\mu_{1} \geq \mu_{2} \geq \ldots \geq \mu_{m}>0\right)$ with $\sum \mu_{i}=n$ or in the form $1^{m_{1}} 2^{m_{2}} \ldots n^{m_{n}}$, where $m_{i}$ is the number of parts $\mu$ which are equal to $i$ (hence $m_{i} \geq 0$ ). Note that $m_{1}+m_{2}+\ldots+m_{n}$ is equal to the number $m$ of non-zero parts of $\mu$.

The symmetric product $\operatorname{Sym}^{n}(S)$ has a natural stratification by locally closed strata labeled by partitions of $n$.

The stratum $\operatorname{Sym}_{\mu}^{n}(S)$ is the set of formal sums of the type $\sum \mu_{i} x_{i}$ (viewed as elements of $\operatorname{Sym}^{n} S$ ) where $x_{i}$ are pairwise distinct. Note that $\operatorname{Sym}_{(1, \ldots, 1)}^{n}$ is a dense open subset of $\operatorname{Sym}^{n}(S)$ and in general $\operatorname{Sym}_{\mu}^{n}(S)$ is isomorphic to a dense open subset of $\operatorname{Sym}^{m_{1}}(S) \times \ldots \times \operatorname{Sym}^{m_{n}}(S)$. A generic element $\left(y_{1}, \ldots, y_{n}\right)$ in the latter product corresponds to the point $y_{1}+2 y_{2}+\ldots+n y_{n}$ in $\operatorname{Sym}_{\mu}^{n}(S)$.

Let $\pi: Z \rightarrow Y$ be a proper projective morphism of algebraic varieties. Suppose that $Y$ decomposes into a finite number of locally closed strata: $Y=\bigcup_{\mu} Y_{\mu}$ and choose an arbitrary point $y_{\mu} \in Y_{\mu}$. Assume that the restriction $\pi: \pi^{-1}\left(Y_{\mu}\right) \rightarrow Y_{\mu}$ is a topological fiber bundle with fiber $\pi^{-1}\left(y_{\mu}\right)$.

Definition (cf. [4] or [7, Chapter 8]). The map $\pi$ is called strictly semi-small if it satisfies

$$
2 \operatorname{dim} \pi^{-1}\left(y_{\mu}\right)=\operatorname{codim} Y_{\mu}
$$

for any stratum $Y_{\mu}$.

The following proposition is an immediate consequence of results of Briançon and Iarrobino (cf. [5], [18]).

Proposition 3.1 (cf. [14]).

The morphism $a_{1}: \operatorname{Hilb}^{n}(S) \rightarrow \operatorname{Sym}^{n}(S)$ is strictly semi-small, with respect to the stratification given by $\operatorname{Sym}_{\mu}^{n}(S)$.

We will give a generalization of this proposition to the case of arbitrary rank. Exactly as in [14] this will lead to some formulas for Poincaré polynomials. These formulas will be later interpreted in representationtheoretic terms. 
The first step is to stratify $M^{U}(r, n)$ appropriately since the natural strata coming from the definition of $M^{U}(r, n)$ are too big. The new finer strata will be labeled by pairs $(s, \mu)$ where $s<n$ is a non-negative integer such that $N(r, n-s)$ is non-empty, and $\mu=\left(\mu_{1} \geq \mu_{2} \geq \ldots \geq\right.$ $\left.\mu_{m} \geq 0\right)$ is a partition of $s$. For each pair $(s, \mu)$ we consider $M_{s, \mu}^{U}(r, n)=$ $N(r, n-s) \times \operatorname{Sym}_{\mu}^{n}(S)$ which is naturally a locally closed subset of $M^{U}(r, n)$. Of course, this is nothing but a "common refinement" of the natural partition of $M^{U}(r, n)$ and the above partition above of the symmetric product.

Part (b) of the next theorem was originally conjectured by V. Ginzburg. It provides a starting point for our generalization of Nakajima's construction.

Proposition 3.2. Let $s$ and $\mu=\left(\mu_{1} \geq \mu_{2} \geq \ldots \geq \mu_{m}>0\right)$ be as above and let $M_{s, \mu}^{U}(r, n)$ be the stratum associated to the pair $(s, \mu)$. Then:

(a) For any point $x_{s, \mu} \in M_{s, \mu}^{U}(r, n)$ the dimension of the fiber $a_{r}^{-1}\left(x_{s, \mu}\right)$ is equal to $(r s-m)$ where $m$ is the number of non-zero parts of $\mu$.

(b) The morphism $M^{G}(r, n) \rightarrow M^{U}(r, n)$ is strictly semi-small with respect ot the stratification given by $M_{s, \mu}^{U}(r, n)$.

Proof. To prove (a) note that by Proposition 2.1 the fiber is isomorphic to $\prod$ Quot $\left(r, m_{i}\right)$ and by Theorem 2.2 its dimension is equal to $\sum_{i=1}^{m}\left(r \mu_{i}-1\right)=r\left(\sum_{i=1}^{m} \mu_{i}\right)-m=r s-m$.

To prove (b) we have to show that $\operatorname{codim} M_{s, \mu}^{U}=2 \operatorname{dim} a_{r}^{-1}\left(x_{s, \mu}\right)=$ $2(r s-m)$. In fact, codim $M_{s, \mu}^{U}(r, n)=\operatorname{dim} N(r, n)-\operatorname{dim} N(r, n-s)-$ $\operatorname{dim} \operatorname{Sym}_{\mu}^{n}(S)=2 r s-2 m=2(r s-m)$. q.e.d.

The semi-smallness result will allow us to relate homological invariants of $M^{G}(r, n)$ and $M^{U}(r, n)$. Recall (cf. [3]) that for any algebraic variety $X$, there exists a remarkable complex of sheaves $I C_{X}$ (intersection cohomology complex) such that its cohomology groups $I H^{*}(X)=H^{*}\left(X, I C_{X}\right)$ (intersection or Goresky-MacPherson homology) satisfy Poincaré duality. If $X$ is a smooth algebraic variety (such as $M^{G}(r, n)$ ) or a quotient of a smooth variety by a finite group action $\left(\right.$ such as $\operatorname{Sym}^{n}(S)$ ) then up to shift $I C_{X}$ is just the constant sheaf $\mathbb{C}$ (cf. [3] of [14]).

We will need a simplified version of Borho-MacPherson formula for the direct image of the intersection homology complex under a projective semismall morphism. This formula is a direct application of 
the Decomposition Theorem due to Beilinson-Berstein-Deligne-Gabber (cf. [3]).

Proposition 3.3. Suppose that a projective morphism $Z \rightarrow Y$ of algebraic varieties is strictly semi-small with respect to some stratification $Y=\bigcup Y_{\mu}$. Suppose further that for any point $y \in Y$ the fiber $\pi^{-1}(y)$ is irreducible. Then

$$
\pi_{*}\left(I C_{Z}\right)=\bigoplus_{\mu} I C_{\bar{Y}_{\mu}}
$$

Corollary 3.4. One has the following direct sum decomposition in the derived category of complexes of sheaves:

$$
\left(a_{r}\right)_{*} I C_{M^{G}(r, n)}=\bigoplus_{s \in\{0, \ldots n\}, \mu \in P(s)} I C_{\bar{M}_{s, \mu}^{U}(r, n)}
$$

where $P(s)$ is the set of all partitions of $s$ and $I C\left(\bar{M}_{s, \mu}^{U}\right)$ denotes the $I C$-complex on the closure of the stratum $M_{s, \mu}^{U} \subset M^{U}(r, n)$.

We intend to use the formula above by taking the cohomology of both sides. The $I C$-complexes supported on the closures of the smaller strata can be understood with the help of the following

Proposition 3.5. Let $(s, \mu)$ and $m_{i}$ be as above and denote by $\operatorname{Sym}^{\mu}(S)$ the direct product $\operatorname{Sym}^{m_{1}}(S) \times \ldots \times \operatorname{Sym}^{m_{n}}(S)$. Then there exists a finite birational morphism respecting the induced stratifications:

$$
\pi_{n, s, \mu}: M^{U}(r, n-s) \times \operatorname{Sym}^{\mu}(S) \rightarrow \bar{M}_{s, \mu}^{U}(r, n)
$$

Proof. Left as an exercise to the reader. q.e.d.

Now [14, Lemma 1] implies that in this situation one can deduce a

Corollary 3.6. In the notation of the previous proposition, one has the following equality in the derived category of sheaves:

$$
\left(\pi_{n, s, \mu}\right)_{*}\left(I C_{M^{U}(r, n-s) \times \operatorname{Sym}^{\mu}(S)}\right)=I C_{\bar{M}_{s, \mu}^{U}(r, n)} .
$$

Recall that intersection homology complex of a space $X$ is defined in such a way that it has non-trivial (global) hypercohomology in the range between $(-k)$ and $k$ where $2 k$ is the real dimension of $X$. 
Definition. The shifted intersection homology Poincaré polynomial $P_{t}(X)$ is defined by the formula

$$
P_{t}(X):=\sum_{-\operatorname{dim}_{\mathbb{C}} X}^{\operatorname{dim}_{\mathbb{C}} X}\left(\operatorname{dim}_{\mathbb{C}} H^{i}\left(X, I C_{X}\right)\right) \cdot t^{i}
$$

Note that for a smooth $X$ this is just the usual Poincaré polynomial multiplied by $t^{-\operatorname{dim}_{\mathbb{C}} X}$.

Theorem 3.7. One has the following identity between Poincaré polynomials of $M^{G}$ and $M^{U}$ :

$$
\frac{\sum_{n=0}^{\infty} q^{n} P_{t}\left(M^{G}(r, n)\right)}{\sum_{n=0}^{\infty} q^{n} P_{t}\left(M^{U}(r, n)\right)}=\prod_{l=1}^{\infty} \frac{\left(1+t^{-1} q^{l}\right)^{b_{1}(S)}\left(1+t^{1} q^{l}\right)^{b_{3}(S)}}{\left(1-t^{-2} q^{l}\right)^{b_{0}(S)}\left(1-q^{l}\right)^{b_{2}(S)}\left(1-t^{2} q^{l}\right)^{b_{4}(S)}}
$$

In short, the series for $M^{G}$ is obtained from the series for $M^{U}$ when multiplied by the Göttsche's formula.

Proof. Following Nakajima's notations, denote by $a_{m}(t)$ the (shifted) Poincaré polynomial $P_{t}\left(\operatorname{Sym}^{m}(S)\right)$. Then a formula due to MacDonald [23] says that

$$
\sum_{m=0}^{\infty} q^{m} a_{m}(t)=\frac{\left(1+t^{-1} q\right)^{b_{1}(S)}(1+t q)^{b_{3}(S)}}{\left(1-t^{-2} q\right)^{b_{0}(X)}(1-q)^{b_{2}(S)}\left(1-t^{2} q\right)^{b_{4}(S)}}
$$

Taking the cohomology of both sides in the formula of Corollary 3.4 and using Corollary 3.6 one obtains:

$$
\begin{aligned}
\sum_{k=0}^{\infty} q^{k} P_{t}\left(M^{G}(r, k)\right) & =\sum_{k=0}^{\infty} \sum_{s=0}^{k} \sum_{\mu \in P(s)} q^{k} P_{t}\left(M^{U}(r, k-s)\right) \cdot P_{t}\left(\operatorname{Sym}^{\mu} S\right) \\
& =\sum_{k=0}^{\infty} \sum_{\substack{0 \leq s \leq k \\
\mu \in P(s)}} q^{k-s} P_{t}\left(M^{U}(r, k-s)\right) \cdot q^{s} P_{t}\left(\operatorname{Sym}^{\mu} S\right) \\
& =\left(\sum_{n=0}^{\infty} q^{n} P_{t}\left(M^{U}(r, n)\right)\right) \cdot\left(\sum_{\substack{s \geq 0 \\
\mu \in P(s)}} q^{s} P_{t}\left(\operatorname{Sym}^{\mu} S\right)\right) .
\end{aligned}
$$


Represent all partitions $\mu$ in the formula above as $\left(1^{m_{1}}, 2^{m_{2}}, \ldots, s^{m_{s}}\right)$. Then

$$
\begin{aligned}
\sum_{\substack{s \geq 0 \\
\mu \in \bar{P}(s)}} q^{s} P_{t}\left(\operatorname{Sym}^{\mu} S\right) & =\sum_{\substack{s \geq 0 \\
\mu \in \bar{P}(s)}} a_{m_{1}}(t)(q)^{m_{1}} a_{m_{2}}(t)\left(q^{2}\right)^{m_{2}} \ldots a_{m_{s}}(t)\left(q^{s}\right)^{m_{s}} \\
& \left.=\prod_{\substack{l=1 \\
\infty}}^{\infty} \sum_{m=0}^{\infty} a_{m}(t)\left(q^{l}\right)^{m}\right) \\
& =\prod_{l=1}^{\infty} \frac{\left(1+t^{-1} q^{l}\right)^{b_{1}(S)}\left(1+t^{1} q^{l}\right)^{b_{3}(S)}}{\left(1-t^{-2} q^{l}\right)^{b_{0}(S)}\left(1-q^{l}\right)^{b_{2}(S)}\left(1-t^{2} q^{l}\right)^{b_{4}(S)}}
\end{aligned}
$$

q.e.d.

\section{Remarks.}

(1) A very similar formula was discovered by Göttsche in [13]. He computes the ratio of generating functions for $M^{G}(r, n)$ and $N(r, n)$ and obtains a similar product (involving more factors).

(2) In [29] $\mathrm{Li}$ and Qin derive a formula relating cohomology of Gieseker spaces for $S$ and its blowup $\widetilde{S}$. Applying Theorem 3.7 we get a relation between the intersection homology of Uhlenbeck compactifications for $S$ and $\widetilde{S}$. This formula is different form the relation between usual homology of $M^{U}(r, n)$ for $S, \widetilde{S}$ (also found by Li and Qin), since Uhlenbeck compactifications are quite singular hence their intersection homology is different from the ordinary (co)homology.

(3) Since $S$ is compact, one of course has $b_{0}=b_{4}=1$ and $b_{1}=b_{3}$. However, Göttsche-Soergel's approach is valid for Hilbert schemes of any quasi-projective surface. It is interesting to know if the computaion above can be extended to the quasi-projective case. For example, one can consider $S=\mathbb{C}^{2}$ viewed as an open subset of $\mathbb{C P}^{2}$ and work with the moduli spaces of sheaves on $\mathbb{C P}^{2}$ equipped with a trivialization on the infinite line $l=\mathbb{C P}^{2} \backslash \mathbb{C}^{2}$. In this particular example the argument used in the proof of the theorem carries over without any changes, but the general case remains open.

Following $[30,1.13]$ one can define pure Hodge structure on the intersection homology of any complex algebraic variety $X$. Then, similarly to the usual shifted Poincaré polynomial, one defines a (shifted) Hodge polynomial $P_{x, y}(X)$ using the pure Hodge structure on $I H^{*}(X)$. When $X$ is smooth this coincides with the usual Hodge polynomial multiplied by $(x y)^{-\operatorname{dim}_{\mathbb{C}} X / 2}$. However, in general it will not be a shift of the virtual Hodge polynomial of $X$. 
Using Saito's theory of mixed Hodge modules (cf. [30]) and the formula for Hodge polynomials of symmetric powers (cf. [6]) one can repeat the arguments above to prove the following

\section{Proposition 3.8.}

(a) There exists an isomorpshism of pure Hodge structures:

$$
I H^{*}\left(M^{G}(r, n)\right) \simeq \bigoplus_{\substack{s \geq 0 \\ \mu \in \bar{P}(s)}} I H^{*}\left(M^{U}(r, n-s)\right) \otimes I H^{*}\left(\operatorname{Sym}^{\mu}(S)\right)
$$

(b) One has the following identity between the intersection homology Hodge polynomials of $M^{G}(r, n)$ and $M^{U}(r, n)$ :

$$
\begin{aligned}
& \sum_{n \geq 0} P_{x, y}\left(M^{G}(r, n)\right) q^{n} \\
& \sum_{n \geq 0} P_{x, y}\left(M^{U}(r, n)\right) q^{n} \\
& \quad=\prod_{l \geq 0} \frac{\left(1+x^{-1} q^{l}\right)^{h^{1,0}}\left(1+y^{-1} q^{l}\right)^{h^{0,1}}\left(1+x q^{l}\right)^{h^{2,1}}\left(1+y q^{l}\right)^{h^{1,2}}}{\left(1-(x y)^{-1} q^{l}\right)^{h^{0,0}}\left(1-x y^{-1} q^{l}\right)^{h^{2,0}}\left(1-q^{l}\right)^{h^{1,1}}\left(1-x^{-1} y q^{l}\right)^{h^{0,2}}\left(1-x y q^{l}\right)^{h^{2,2}}}
\end{aligned}
$$

where $h^{r, s}$ are the (usual) Hodge numbers of $S$.

\section{Correspondences}

In this section we give a very natural generalization of correspondences used by Nakajima and Grojnowski and prove that their action on the cohomology satisfies the correct commutation relations. Of course, as one might guess from the formula of Theorem 3.7, the corresponding module over the oscillator algebra is not irreducible any more. In fact, the space of vacuum vectors can be naturally identified with intersection homology of Uhlenbeck compactifications.

We now briefly recall the definition of the convolution product, cf. [7]. Let $M_{1}, M_{2}$ be two smooth projective varieties and $Z$ be a subvariety of $M_{1} \times M_{2}$, such that restrictions of the two projections

$p_{1}: Z \rightarrow M_{1}, p_{2}: Z \rightarrow M_{2}$ are proper. Such $Z$ defines the map $P_{Z}: H^{*}\left(M_{1}\right) \rightarrow H^{*}\left(M_{2}\right)$ by the formula

$$
P_{Z}(\alpha)=\left(p_{2}\right)_{*}\left(p_{1}^{*} \alpha\right),
$$


More generally, let $\beta \in H^{*}(Z)$ be a cohomology class (in an appropriate cohomology theory if $Z$ is singular). Then we can consider the convolution operator $P_{Z}^{\beta}: H^{*}\left(M_{1}\right) \rightarrow H^{*}\left(M_{2}\right)$ defined by

$$
P_{Z}^{\beta}(\alpha)=\left(p_{2}\right)_{*}\left(\beta \cup p_{1}^{*} \alpha\right) .
$$

Of course, we can switch the roles of $M_{1}$ and $M_{2}$ and define the map in the other direction.

If $M_{3}$ is a third smooth projective variety, $Z^{\prime} \subset M_{2} \times M_{3}$ is a subvariety which projects properly to $M_{2}$ and $M_{3}$, and $\beta^{\prime} \in H^{*}\left(Z^{\prime}\right)$ then the composition $P_{Z^{\prime}}^{\beta^{\prime}} \circ P_{Z}^{\beta}: H^{*}\left(M_{1}\right) \rightarrow H^{*}\left(M_{3}\right)$ can be described as follows. Consider the subvariety $\widetilde{Z} \subset M_{1} \times M_{3}$ given by

$$
\widetilde{Z}=p_{13}\left(p_{12}^{-1}(Z) \cap p_{13}^{-1}\left(Z^{\prime}\right)\right),
$$

where $p_{12}$ is the projections $M_{1} \times M_{2} \times M_{3} \rightarrow M_{1} \times M_{2}$, and similarly for $p_{13}$ and $p_{23}$. We define the cohomology class $\widetilde{\beta} \in H^{*}(\widetilde{Z})$ as $\left(p_{13}\right)_{*}\left(p_{12}^{*}(\beta) \cup p_{23}^{*}\left(\beta^{\prime}\right)\right)$. Then, (cf. [7, Chapter 3$\left.]\right)$, we have the equality

$$
P_{Z^{\prime}}^{\beta^{\prime}} \circ P_{Z}^{\beta}=P_{\widetilde{Z}}^{\widetilde{\beta}}: H^{*}\left(M_{1}\right) \rightarrow H^{*}\left(M_{3}\right)
$$

We now describe the subvarieties in the products of the moduli spaces, to which the above convolution construction will be applied.

Definition. For $i>0$, define a subvariety

$$
P_{-i} \subset \coprod_{n} M^{G}(r, n) \times M(r, n+i)
$$

as a set of all pairs $\left(\mathcal{F}_{1}, \mathcal{F}_{2}\right)$ such that:

(1) $\mathcal{F}_{1}^{* *} \simeq \mathcal{F}_{2}^{* *}$

(2) $\mathcal{F}_{2} \subset \mathcal{F}_{1}$ as subsheaves of the common double dual;

(3) $\operatorname{Supp}\left(\mathcal{F}_{1} / \mathcal{F}_{2}\right)=\{x\}$ for some $x \in X$.

Similarly, we define $P_{i} \subset \coprod_{n} M^{G}(r, n+i) \times M(r, n)$ for $i>0$ by exchanging the roles of $\mathcal{F}_{1}$ and $\mathcal{F}_{2}$.

Remark. Since $\mathcal{F}_{1}$ and $\mathcal{F}_{2}$ are stable, so is $\mathcal{F}:=\mathcal{F}_{1}^{* *} \simeq \mathcal{F}_{2}^{* *}$. This implies $\operatorname{Hom}(\mathcal{F}, \mathcal{F})=\mathbb{C}$, and the embeddings $\mathcal{F}_{1} \hookrightarrow \mathcal{F}, \mathcal{F}_{2} \hookrightarrow \mathcal{F}$ are defined canonically up to multiplication by a scalar. This ensures that the second condition is well-defined. 
One has a natural morphism $\Pi: P_{i} \rightarrow S$ given by

$$
\Pi\left(\mathcal{F}_{1}, \mathcal{F}_{2}\right)=x \quad \text { for }\left(\mathcal{F}_{1}, \mathcal{F}_{2}\right) \in P_{i}
$$

where $x$ is the unique element of $\operatorname{Supp}\left(\mathcal{F}_{1} / \mathcal{F}_{2}\right)$. One can give a rigorous definition of $\Pi$ using universal sheaves on moduli spaces and [17, Example 4.3.6] but we will not do it here.

Denote the dimension of $M^{G}(r, n)$ by $2 r n+a$ where $a$ is a number depending on $S, r, L$ and $H$ but not on $n$, cf. (2). Then the dimension of $P_{-i} \cap M^{G}(n-i) \times M^{G}(n)$ is equal to $2 r n+a-r i+1$ (this follows from Nakajima's argument [27, 8.3] and Theorem 2.2).

Let $P_{ \pm i}^{\alpha}: \bigoplus_{n} H^{*}\left(M^{G}(r, n)\right) \rightarrow \bigoplus_{n} H^{*}\left(M^{G}(r, n \mp i)\right)$, for $\alpha \in H^{*}(S)$ be the convolution operators induced by the classes $\Pi^{*}(\alpha) \in H^{*}\left(P_{ \pm i}\right)$. Computing the dimensions we can see that $P_{-i}^{\alpha}$ increases the cohomological degree by $2(r i-1)+\operatorname{deg} \alpha$.

The next theorem asserts that $\bigoplus_{n} H^{*}\left(M^{G}(r, n)\right)$ becomes a representation space of the Heisenberg/Clifford algebra generated by $P_{i}^{\alpha}$ and $P_{-i}^{\beta} /(-1)^{r i-1} r$. It is a direct generalization of [27, Theorem 8.13].

Theorem 4.1. The following relations hold:

$$
\begin{array}{rlrl}
{\left[P_{i}^{\alpha}, P_{j}^{\beta}\right]} & =(-1)^{r i-1} \text { ri } \delta_{i+j, 0}\langle\alpha, \beta\rangle I d & & \text { if } \quad(-1)^{\operatorname{deg} \alpha \cdot \operatorname{deg} \beta}=1, \\
\left\{P_{i}^{\alpha}, P_{j}^{\beta}\right\} & =(-1)^{r i-1} \text { ri } \delta_{i+j, 0}\langle\alpha, \beta\rangle I d & \text { otherwise, }
\end{array}
$$

where $\langle\alpha, \beta\rangle$ is the intersection form on $S,[A, B]=A B-B A$ and $\{A, B\}=A B+B A$.

Exactly as in [27, Corollary 8.16] we deduce:

Corollary 4.2. The cohomology groups $\oplus_{n=0}^{\infty} H^{*}\left(M^{G}(r, n)\right)$ form a tensor product of an irreducible highest weight representation of the oscillator algebra, with a trivial representation on the intersection homology groups of Uhlenbeck compactifications.

Example. Let $r=2, i=1, S=\mathbb{P}^{2}$ and $L \simeq H \simeq \mathcal{O}(1)$. Choose $\alpha$ to be the fundamental class and $\beta$ the class of a point $x \in \mathbb{P}^{2}$. Then the formula above predicts that $\left[P_{1}^{\alpha}, P_{-1}^{\beta}\right]=(-2) I d$. This can be seen directly. 
It is known that $M^{G}(2,1)$ is a single point corresponding to the twist $T_{\mathbb{P}^{2}}(-1)$ of the tangent bundle (cf. [28, 3.2]). The Uhlenbeck compactification $M^{U}(2,2)$ can be described as follows. Let $V$ be the 6dimensional space of symmetric $3 \times 3$ matrices. Then $M^{U}(2,2)$ can be identified with a hypersurface $H$ in $P(V)$ correspoding to all rank $\leq 2$ symmetric matrices (cf. $[28,4.3])$. Its singular locus $P \simeq \mathbb{P}^{2}$ corresponds to symmetric matrices of rank one. It can be deduced from (cf. [28, 4.3]) that $M^{G}(2,2)$ is isomorphic to the blowup of $P$.

Let $D \subset M^{G}(2,2)$ be the exceptional divisor. Each one-dimensional fiber of $D \rightarrow P$ corresponds to the set of sheaves $\mathcal{F}$ which fit a short exact sequence

$$
0 \rightarrow \mathcal{F} \rightarrow T_{\mathbb{P}^{2}}(-1) \rightarrow \mathbb{C}_{x} \rightarrow 0,
$$

where $x \in \mathbb{P}^{2} \simeq P$. Let $l$ be such a fiber and $[p t]$ the generator of $H^{0}\left(M^{G}(2,1)\right)$. Since $P_{1}^{\alpha}([p t])=0$ and $P_{-1}^{\beta}([p t])=[l]$ we expect that $P_{1}^{\alpha}([l])=-2[p t]$. It follows from definitions that this is equivalent to $[D] \cdot[l]=-2$. The last equality is true since $H$ has an $A_{2}$-singularity at any point of $P$.

Now we begin to prove Theorem 4.1. Most of Nakajima's proof, cf. [27, Chapter 8], can be repeated word-by-word with almost no changes. In particular, we have the following result:

Theorem 4.3. For any $v \in H^{*}\left(M^{G}(r, n)\right)$, the following relations hold:

$$
\begin{array}{rlrl}
{\left[P_{i}^{\alpha}, P_{j}^{\beta}\right](v)} & =c_{i, n} \delta_{i+j, 0}\langle\alpha, \beta\rangle v & \text { if } \quad(-1)^{\operatorname{deg} \alpha \cdot \operatorname{deg} \beta}=1, \\
\left\{P_{i}^{\alpha}, P_{j}^{\beta}\right\}(v) & =c_{i, n} \delta_{i+j, 0}\langle\alpha, \beta\rangle v & & \text { otherwise, }
\end{array}
$$

where $\langle\alpha, \beta\rangle$ is the intersection form on $H^{*}(S)$ and $c_{i, n}$ is some constant depending apriori on $i$ and $n$ but not on the classes $\alpha$ and $\beta$.

The proof is a mere repetition of the argument presented in $[27,8.4]$ (where all the dimension statements are proved using Theorem 2.2).

Lemma 4.4. For each $i$, the constants $c_{i, n}$ are independent of $n$.

Proof. Let $k \neq \pm i$. By Theorem 4.3 we have $\left[P_{-k}^{\gamma},\left[P_{i}^{\alpha}, P_{-i}^{\beta}\right]\right]=0$. Take $v \in H^{*}\left(M^{G}(r, n)\right)$. Applying the double commutator to $v$, one gets

$$
\langle\alpha, \beta\rangle\left(c_{i, n}-c_{i, n+k}\right) P_{-k}^{\gamma} v=0 .
$$

One can choose $v, \alpha, \beta, \gamma$ in such a way that $\langle\alpha, \beta\rangle \neq 0$ and $P_{\gamma}[-k](v) \neq$ 0 . Therefore, one has $c_{i, n}=c_{i, n+k}$ if $k \neq \pm i$. If $i \neq \pm 1$ we can take 
$k=1$ and obtain $c_{i, n}=c_{i, n+1}$. If $i= \pm 1$ we take $k=2,3$ and get $c_{1, n}=c_{1, n+2}=c_{1, n+3}$ which also implies that $c_{1, n}$ is independent of $n$. q.e.d.

In view of this lemma we will be writing $c_{i}$ instead of $c_{i, n}$.

Recall the general setup of Nakajima's Chapter 9 (or rather its adaptation to our case). We choose and fix two smooth transversal curves $C$ and $C^{\prime}$ in one very ample linear system on $S$. Take such an $s$ that $M^{G}(r, s)$ is non-empty and fix a locally free sheaf $\mathcal{E}$ correspoding to a point in $M^{G}(r, s)$.

We will compute numbers $c_{i}$ using the formalism of generating functions and a connection with symmetric functions discovered by Nakajima. The actual idea of using embedded curves is originally due to Grojnowski.

To that end, consider the subvariety $L^{k, s} \subset M^{G}(r, n)$ formed by all sheaves $\mathcal{F}$ such that:

(a) $\mathcal{F}^{* *} \simeq \mathcal{E}$

(b) The quotient $A_{\mathcal{F}}:=\mathcal{E} / \mathcal{F}$ is an Artin sheaf of length $k=n-s$ supported at finitely many points of $C$.

Definition. For each partition $\mu=\left(\mu_{1} \geq \ldots \geq \mu_{l}>0\right)$ of $k$ let $\left(L^{\mu, s}\right)^{\circ}$ be the set of all sheaves $\mathcal{F}$ such that:

(a) $\mathcal{F}^{* *} \simeq \mathcal{E}$

(b) The quotient $A_{\mathcal{F}}=\mathcal{E} / \mathcal{F}$ is supported at pairwise distinct points $x_{j} \in C, 1 \leq j \leq l$ with multiplicities $\mu_{j}$.

Denote also by $L^{\mu, s}$ the closure of $\left(L^{\mu, s}\right)^{\circ}$.

Definition. Similarly, we define $\widehat{L}^{\mu, s}$ to be the closure of the subset of all sheaves $\mathcal{F}$ satisfying:

(a) $\mathcal{F} \subset \mathcal{F}^{\prime}$ for some $\mathcal{F}^{\prime} \in M^{G}(r, s)$;

(b) the quotient $\mathcal{F}^{\prime} / \mathcal{F}$ is supported at pairwise distinct points $x_{j} \in C$, $1 \leq j \leq l$ with multiplicities $\mu_{j}$. 
Proposition 4.5. The closed subvarieties $L^{\mu, s}$ are the irreducible components of $L^{k, s}$. Each component $L^{\mu, s}$ is of dimension rk. Similarly, $\widehat{L}^{\mu, s}$ is the set of all irreducible components of $\widehat{L}^{k, s}$ and all $\widehat{L}^{\mu, s}$ are of the same dimension.

Proof. If we fix all the points $x_{j}$ is the definition of $L^{\mu, s}$ then by Theorem 2.2 the variety of all sheaves with the required condition has dimension $\sum_{i=1}^{l}\left(r \mu_{i}-1\right)=r k-l$. Since the points $x_{j}, j=1, \ldots, l$ are allowed to move in an $l$-dimensional family, we obtain $\operatorname{dim} L^{\mu, s}=r k$. By the irreducibility part of Theorem 2.2 we conclude that each $L^{\mu, s}$ is irreducible.

It follows from the definition of $L^{k, s}$ that it is a union of the closed subsets $L^{\mu, s}$. Hence, $L^{\mu, s}$ are the irreducible components of $L^{k, s}$. q.e.d.

Note that $P_{-i}^{C}$ maps classes of (cohomological) degree $b$ on $M^{G}(r, n)$ to classes of degree $r i+b$ on $M^{G}(r, n+i)$. Thus, the subspace $\bigoplus H^{r n}\left(M^{G}(r, n)\right)$ is preserved by $P_{-i}^{C}$. Moreover, the next theorem shows that in fact even the smaller subspace generated by the classes of $L^{\mu, s}$, is invariant under $P_{-i}^{C}(i>0)$.

Theorem 4.6 (cf. [27, Thm. 9.14]). For $i>0$, we have

$$
P_{-i}^{C}\left[L^{\mu, s}\right]=\sum_{\lambda} a_{\lambda \mu}\left[L^{\lambda, s}\right]
$$

where the summation is over partitions $\lambda$ of $|\mu|+i$ which are obtained as follows:

(a) add $i$ to a term in $\mu$, say $\mu_{j}$ (possibly 0), and then

(b) arrange it in descending order.

The coefficient $a_{\lambda \mu}$ is $\#\left\{l \mid \lambda_{l}=\mu_{j}+i\right\}$.

A similar statement is true for the classes of $\widehat{L}^{\mu, s}$.

This theorem will be proved in Section 5 .

Let $[v a c]$ be the cohomology class of the point $\mathcal{E} \in M^{G}(r, s)$ and $[V a c]$ be the fundamental class of $M^{G}(r, s)$. Theorem 4.6 allows us to establish a connection with the theory of symmetric functions as follows. Let $L$ (resp. $\widehat{L})$ be the subspace in $\bigoplus_{n} H^{*}\left(M^{G}(r, n)\right)$ generated by the classes of $L^{\mu, s}$ and $[v a c]$ (resp. $\widehat{L}^{\mu, s}$ and $[V a c]$ ). We define a $\mathbb{C}$-linear 
isomorphism from $L$ (resp. $\widehat{L}$ ) onto the space $\Lambda$ of symmetric functions in infinitely many variables (cf. [27, 9.1], [24]). This isomorphism sends [vac] (resp. $[V a c])$ to $1 \in \Lambda$ and $\left[L^{\mu, s}\right]$ (resp. $\left[\widehat{L}^{\mu, s}\right]$ ) to the orbit sum function $m_{\lambda}$ (cf. loc. cit.).

Theorem 4.6 means that the operator $P_{-i}^{C}$ corresponds under the isomorphism above to multiplication by the $i$-th power sum (or Newton function) $p_{i} \in \Lambda$ (loc. cit.). This provides us with several identities between cohomology classes coming from classical identities between symmetric functions.

Note that for $\mu=\left(1^{k}\right)$ the corresponding variety $L^{\mu, s}$ is isomorphic to the global Quot scheme $\operatorname{Quot}_{C}^{k}(\mathcal{E})$ on the curve $C$. This scheme parametrizes quotient sheaves $\left.\mathcal{E}\right|_{C} \rightarrow A$ on $C$, where $A$ is of length $k$. Every such quotient on $C$ defines a sheaf on $S$, namely the kernel of the composition $\left.\mathcal{E} \rightarrow \mathcal{E}\right|_{C} \rightarrow A$. The class of $\operatorname{Quot}_{C}^{k}(\mathcal{E})$ corresponds to the $k$-th elementary symmetric function $e_{k}=m_{\left(1^{k}\right)}$.

Similarly, we will slightly abuse notation by writing $\operatorname{Quot}_{C}^{k}(s)$ instead of $\widehat{L}^{\left(1^{k}\right), s}$. This variety is a birational image of the family of schemes $Q u o t_{C}^{k}\left(\mathcal{F}^{\prime}\right)$ parametrized by points $\mathcal{F}^{\prime}$ of $M^{G}(r, s)$.

Repeating the arguments of Nakajima, (see [27, formula 9.16]) we obtain the formulas

$$
\begin{aligned}
\sum_{n=0}^{\infty} z^{n}\left[\operatorname{Quot}_{C}^{n}(\mathcal{E})\right] & =\exp \left(\sum_{i=1}^{\infty} \frac{z^{i} P_{i}^{C}}{(-1)^{i-1} i}\right) \cdot[v a c] \\
\sum_{n=0}^{\infty} z^{n}\left[\operatorname{Quot}_{C}^{n}(s)\right] & =\exp \left(\sum_{i=1}^{\infty} \frac{z^{i} P_{i}^{C}}{(-1)^{i-1} i}\right) \cdot[\text { Vac }] .
\end{aligned}
$$

These formulas arise from the classical identity between Newton symmetric functions and elementary symmetric functions (cf. [24]). They are the first ingredient in our computation of $c_{i}$. The second ingredient is provided by

Theorem 4.7 (cf. [27, Exercise 9.23]). The following relation holds:

$$
\sum_{n=0}^{\infty} z^{2 n}\left\langle Q u o t_{C}^{n}(\mathcal{E}), \operatorname{Quot}_{C^{\prime}}^{n}(s)\right\rangle=\left(1-(-1)^{r} z^{2}\right)^{r\left\langle C, C^{\prime}\right\rangle}
$$

The proof of this theorem will be given in Section 6 for the case when the intersection $C \bigcap C^{\prime}$ is transversal (which is sufficient for our purposes). Then the general case can be deduced from Theorem 4.1 via the argument of the next section. 
End of the proof of Theorem 4.1. Following Nakajima, we introduce the following notations:

$$
C_{-}(z)=\sum_{i=1}^{\infty} \frac{P_{-i}^{C} z^{i}}{(-1)^{i-1} i} ; \quad C_{+}(z)=\sum_{i=1}^{\infty} \frac{P_{i}^{C} z^{i}}{(-1)^{i-1} i} .
$$

Note that $C_{-}(z)$ is adjoint to $C_{+}(z)$ with respect to the intersection form on the direct sum $\bigoplus_{n} H^{*}\left(M^{G}(r, n)\right)$ (the cohomology groups for different $n$ are orthogonal to each other). We extend this form to power series in $z$ by $z$-linearity. By Theorem 4.7 and (3), (4) one has:

$$
\begin{aligned}
\left(1-(-1)^{r} z^{2}\right)^{r\left\langle C, C^{\prime}\right\rangle} & =\sum_{n=0}^{\infty} z^{2 n}\left\langle\operatorname{Quot}_{C}^{n}(\mathcal{E}), \operatorname{Quot}_{C^{\prime}}^{n}(s)\right\rangle \\
& =\left\langle\sum_{n=0}^{\infty} z^{n} \operatorname{Quot}_{C}^{n}(\mathcal{E}), \sum_{n=0}^{\infty} z^{n} \operatorname{Quot}_{C^{\prime}}^{n}(s)\right\rangle \\
& =\left\langle\exp \left(C_{-}(z)\right) \cdot[v a c], \exp \left(C_{-}^{\prime}(z)\right) \cdot[\text { Vac }]\right\rangle \\
& =\left\langle\exp \left(C_{+}^{\prime}(z)\right) \exp \left(C_{-}(z)\right) \cdot[v a c],[\text { Vac }]\right\rangle
\end{aligned}
$$

where the last equation follows from adjointness.

We will use the following identity which holds for any pair of operators $A$ and $B$ :

$\exp (-A) B \exp (A)=\exp (-a d A)(B)=1-(a d A)(B)+\frac{(a d A)^{2}}{2 !}(B)-\ldots$

Putting $A=C_{-}(z), B=\exp \left(C_{+}^{\prime}(z)\right)$ we obtain

$$
\begin{aligned}
& \left\langle\exp \left(C_{+}^{\prime}(z)\right) \exp \left(C_{-}(z)\right) \cdot[v a c],[V a c]\right\rangle= \\
& \quad=\left\langle\exp \left(C_{-}(z)\right)\left[\exp \left(-a d C_{-}(z)\right)\left(\exp \left(C_{+}^{\prime}(z)\right)\right)\right] \cdot[v a c],[V a c]\right\rangle .
\end{aligned}
$$

An explicit computation shows that

$$
\left[C_{-}(z), \exp \left(C_{+}^{\prime}(z)\right)\right]=-\left(\sum_{n=1}^{\infty} \frac{c_{n}}{n^{2}}\left\langle C, C^{\prime}\right\rangle z^{2 n}\right) \exp \left(C_{+}^{\prime}(z)\right) .
$$

Denote the expression $\sum_{n=1}^{\infty} \frac{c_{n}}{n^{2}}\left\langle C, C^{\prime}\right\rangle z^{2 n}$ by $\Phi(z)$. Then by the previous formula:

$$
\exp \left(-a d C_{-}(z)\right)\left(\exp \left(C_{+}^{\prime}(z)\right)\right)=\exp (\Phi(z)) \exp \left(C_{+}^{\prime}(z)\right)
$$


Collecting the results of computations, we obtain:

$$
\begin{aligned}
\left(1-(-1)^{r} z^{2}\right)^{r\left\langle C, C^{\prime}\right\rangle} & =\exp (\Phi(z))\left\langle\exp \left(C_{-}(z)\right) \exp \left(C_{+}^{\prime}(z)\right) \cdot[v a c],[\text { Vac }]\right\rangle \\
& =\exp (\Phi(z))\left\langle\exp \left(C_{-}(z)\right) \cdot[v a c],[\text { Vac }]\right\rangle \\
& =\exp (\Phi(z)) .
\end{aligned}
$$

The last equality holds since all $P_{-i}^{C}$ involved in the definition of $C_{+}(z)$, map $[v a c]$ to the orthogonal complement of $[V a c]$. By definition of $\Phi(z)$ we have

$$
\sum_{n=1}^{\infty} \frac{c_{n}}{n^{2}}\left\langle C, C^{\prime}\right\rangle z^{2 n}=r\left\langle C, C^{\prime}\right\rangle \log \left(1-(-1)^{r} z^{2}\right) .
$$

Hence $c_{n}=(-1)^{r n-1} r n$ which completes the proof of Theorem 4.1.

q.e.d.

\section{A transversality result}

In the case $r=1$, Theorem 4.6 has a simple proof (cf. [27]) using local coordinates on $S$. While this result is still true in higher ranks the proof of it requires a more detailed analysis of the geometry of the moduli space to be provided below.

First consider the situation at the set-theoretic level. It follows for the definitions that if $\left(\mathcal{F}_{1}, \mathcal{F}_{2}\right) \in P_{-i}^{C}$ then $\mathcal{F}_{1}^{* *} \simeq \mathcal{F}_{2}^{* *}$ and $\operatorname{Supp}\left(\mathcal{F}_{1} / \mathcal{F}_{2}\right)=$ $\{x\} \in C$. In particular, if $\mathcal{F}_{1} \in L^{\mu, s}$ then $\mathcal{F}_{2}$ necessarily belongs to one of the $L^{\lambda, s}$ where the partition $\lambda$ is as described in the theorem. Hence $P_{-i}^{C}\left[L^{\mu, s}\right]$ is a linear combination of $\left[L^{\lambda, s}\right]$ with integral coefficients.

Our goal is to show that for generic $\mathcal{F}_{2} \in L^{\lambda, s}$ the intersection

$$
p_{1}^{-1}\left(L^{\mu, s}\right) \bigcap P_{-i}^{C} \bigcap p_{2}^{-1}\left(\mathcal{F}_{2}\right) \subset M^{G}(r, s+|\mu|) \times M^{G}(r, s+|\lambda|)
$$

is finite and consists of exactly $a_{\lambda \mu}$ points.

Recall that by definition all sheaves $\mathcal{F}$ representing the points of $L^{\lambda, s}$ satisfy $\mathcal{F}^{* *} \simeq \mathcal{E}$ where $\mathcal{E}$ is a fixed vector bundle. In particular, this holds for $\mathcal{F}_{1} \in L^{\mu, s}$ and $\mathcal{F}_{2} \in L^{\lambda, s}$. Consider the quotient $A_{\mathcal{F}_{2}}=\mathcal{E} / \mathcal{F}_{2}$. If $\lambda=\left(\lambda_{1} \geq \cdots \geq \lambda_{p}>0\right)$ then $A_{\mathcal{F}_{2}}$ is supported at some points $x_{j} \in C$, $1 \leq j \leq p$ with multiplicities $\lambda_{j}$. Therefore one can write $A_{\mathcal{F}_{2}}$ as a direct sum $\bigoplus\left(A_{\mathcal{F}_{2}}\right)_{x_{j}}$.

We claim that for generic $\mathcal{F}_{2}$ there exist local coordinates $\left(\zeta_{j}, \xi_{j}\right)$ at $x_{j}$ such that $\left(A_{\mathcal{F}_{2}}\right)_{x_{j}} \simeq \mathbb{C}\left[\zeta_{j}\right] /\left(\zeta_{j}\right)^{\lambda_{j}}$. 
In fact, choose a trivialization $\mathcal{E} \simeq \mathcal{O}^{\oplus r}$ in the neighbourhood of $x_{j}$ and fix some system of coordinates $\left(\zeta_{j}^{\prime}, \xi_{j}^{\prime}\right)$ centered at $x_{j}$. Consider such quotients $\mathcal{O}^{\oplus r} \rightarrow A$ of length $\lambda_{j}$ at $x_{j}$ that the first component $\mathcal{O} \rightarrow A$ is surjective and its kernel is generated by $m_{x_{j}}^{\lambda_{j}}$ and some element $\xi_{j}=\xi_{j}^{\prime}+\sum_{k=1}^{\lambda_{j}-1} a_{k}\left(\zeta_{j}^{\prime}\right)^{k}$, where $a_{1}, \ldots, a_{\lambda_{j}-1}$ are arbitrary complex numbers. The other components $\mathcal{O}^{\oplus(r-1)} \rightarrow A$ can be chosen arbitrarily. If we take $\zeta_{j}=\zeta_{j}^{\prime}$ then $A$ has the required form in the local coordinates $\left(\zeta_{j}, \xi_{j}\right)$. Thus we obtain a $\left(r \lambda_{j}-1\right)$-dimensional family of non-isomorphic quotients. Here $\lambda_{j}-1$ parameters come from the choice of $\xi_{j}$ and the other $(r-1) \lambda_{j}$ parameters come from the choice of the map $\mathcal{O}^{\oplus(r-1)} \rightarrow$ $A$. By Theorem 2.2, this family forms a dense subset in $Q u o t\left(r, \lambda_{j}\right)$ and this implies our assertion.

Hence we can assume that $\mathcal{E} / \mathcal{F}_{2}$ satisfies the conditions described above. Then a choice of $\mathcal{F}_{1}$ such that $\left(\mathcal{F}_{1}, \mathcal{F}_{2}\right) \in P_{-i}^{C}$ amounts to choosing a $\left(\lambda_{j}-i\right)$-dimensional quotient $\left(A_{\mathcal{F}_{1}}\right)_{x_{j}}$ of $\left(A_{\mathcal{F}_{2}}\right)_{x_{j}} \simeq \mathbb{C}\left[\zeta_{j}\right] /\left(\zeta_{j}\right)^{\lambda_{j}}$. If $x_{j}$ is fixed, this can be done in a unique way. Therefore the number of points in $p_{1}^{-1}\left(L^{\mu, s}\right) \bigcap P_{-i}^{C}$ over a generic $\mathcal{F}_{2}$ is equal to the number of ways to subtract $i$ from one of the parts of partition $\lambda$, and obtain partition $\mu$. This number is exactly $a_{\lambda \mu}$.

To finish the proof of Theorem 4.6 we need to show that $p^{-1}\left(L^{\mu, s}\right)$ intersects $P_{-i}^{C}$ transversely. To that end, we prove the following lemma

Lemma 5.1. Let $\left(\mathcal{F}_{1}, \mathcal{F}_{2}\right) \in P_{-i}^{C} \bigcap M^{G}(r, s+|\mu|) \times M^{G}(r, s+|\lambda|)$ be a smooth point of $P_{-i}^{C}$ and assume that $\mathcal{F}_{1} \in L^{\mu, s}\left(\right.$ resp. $\left.\mathcal{F}_{2} \in L^{\lambda, s}\right)$ is generic in the sense described above. Then the intersection

$$
W=T_{\left(\mathcal{F}_{1}, \mathcal{F}_{2}\right)} p_{1}^{-1}\left(L^{\mu, s}\right) \bigcap T_{\left(\mathcal{F}_{1}, \mathcal{F}_{2}\right)} P_{-i}^{C}
$$

of tangent spaces to $p_{1}^{-1}\left(L^{\mu, s}\right)$ and $P_{-i}^{C}$ projects isomorphically under $p_{2}$ onto the tangent space $T_{\mathcal{F}_{2}}\left(L^{\lambda, s}\right)$.

Proof. It suffices to prove that if $(v, w) \in W$ then $w \in T_{\mathcal{F}_{2}}\left(L^{\lambda, s}\right)$ and $v$ is uniquely defined by $w$. Then the dimension count shows that in fact the map $d p_{2}: W \rightarrow T_{\mathcal{F}_{2}}\left(L^{\lambda, s}\right)$ is an isomorphism. Our proof consists of several steps.

Step 1. First we compute the tangent spaces $T_{\mathcal{F}_{1}}\left(L^{\mu, s}\right)$ and $T_{\mathcal{F}_{2}}\left(L^{\lambda, s}\right)$.

To that end, recall (cf. [1]) that the tangent space $T_{\mathcal{F}_{1}}\left(M^{G}(r, s+|\mu|)\right)$ is isomorphic to the kernel of the natural trace map $\operatorname{tr}^{1}: \operatorname{Ext}_{S}^{1}\left(\mathcal{F}_{1}, \mathcal{F}_{1}\right) \rightarrow$ 
$H^{1}(S, \mathcal{O})$. Infinitesimal deformations of $\mathcal{F}_{1}$ with fixed $\mathcal{E}=\mathcal{F}_{1}^{* *}$ correspond to the subspace $\operatorname{Hom}_{S}\left(\mathcal{F}_{1}, \mathcal{E} / \mathcal{F}_{1}\right) \subset \operatorname{Ext}_{S}^{1}\left(\mathcal{F}_{1}, \mathcal{F}_{1}\right)$. The embedding of this subspace is just the boundary map which comes from applying $\operatorname{Hom}\left(\mathcal{F}_{1}, \cdot\right)$ to a short exact sequence $0 \rightarrow \mathcal{F}_{1} \rightarrow \mathcal{E} \rightarrow \mathcal{E} / \mathcal{F}_{1} \rightarrow 0$.

Since $\mathcal{E} / \mathcal{F}_{1}=A_{\mathcal{F}_{1}}=\bigoplus_{x_{j} \in \operatorname{Supp}\left(A_{\mathcal{F}_{1}}\right)}\left(A_{\mathcal{F}_{1}}\right)_{x_{j}}$, one has a direct sum decomposition

$$
\operatorname{Hom}_{S}\left(\mathcal{F}_{1}, A_{\mathcal{F}_{1}}\right)=\bigoplus_{x_{j} \in \operatorname{Supp}\left(A_{\mathcal{F}_{1}}\right)} \operatorname{Hom}_{S}\left(\mathcal{F}_{1},\left(A_{\mathcal{F}_{1}}\right)_{x_{j}}\right)
$$

Recall that $\mu_{j}=$ mult $_{x_{j}}\left(A_{\mathcal{F}_{1}}\right)$. Suppose that $\mathcal{F}_{1}$ is generic in the sense that it has a local description given in the previous section. Then locally in the neighbourhood of $x_{j} \in S$ we have embeddeings $\mathcal{E}\left(-\mu_{j} C\right) \subset$ $\mathcal{F}_{1} \subset \mathcal{E}$. Therefore the space $\operatorname{Hom}_{S}\left(\mathcal{F}_{1},\left(A_{\mathcal{F}_{1}}\right)_{x_{j}}\right)$ contains a subspace $\operatorname{Hom}_{S}\left(\mathcal{F}_{1} / \mathcal{E}\left(-\mu_{j} C\right),\left(A_{\mathcal{F}_{1}}\right)_{x_{j}}\right)$. Now it is easy to see that the tangent space $T_{\mathcal{F}_{1}}\left(L^{\mu, s}\right)$ corresponds precisely to the subspace

$$
\begin{aligned}
\bigoplus_{x_{j} \in \operatorname{Supp}\left(A_{\mathcal{F}_{1}}\right)} \operatorname{Hom}_{S}\left(\mathcal{F}_{1} / \mathcal{E}\left(-\mu_{j} C\right),\left(A_{\mathcal{F}_{1}}\right)_{x_{j}}\right) & \subset \operatorname{Hom}_{S}\left(\mathcal{F}_{1}, A_{\mathcal{F}_{1}}\right) \\
& \subset \operatorname{Ext}_{S}^{1}\left(\mathcal{F}_{1}, \mathcal{F}_{1}\right) .
\end{aligned}
$$

Similar computation applies to $\mathcal{F}_{2} \in L^{\lambda, s}$.

Step 2. Now we assume that

$$
\left(\mathcal{F}_{1}, \mathcal{F}_{2}\right) \in P_{-i}^{C} \subset M^{G}(r, s+|\mu|) \times M^{G}(r, s+|\lambda|)
$$

is a generic point. We want to describe the tangent space to $P_{-i}^{C}$ at the point $\left(\mathcal{F}_{1}, \mathcal{F}_{2}\right)$, as a subspace of $\operatorname{Ext}_{S}^{1}\left(\mathcal{F}_{1}, \mathcal{F}_{1}\right) \oplus \operatorname{Ext}_{S}^{1}\left(\mathcal{F}_{2}, \mathcal{F}_{2}\right)$.

Recall that the vectors in $\operatorname{Ext}_{S}^{1}\left(\mathcal{F}_{1}, \mathcal{F}_{1}\right)$ correspond to extensions $0 \rightarrow \mathcal{F}_{1} \rightarrow \mathcal{G}_{1} \rightarrow \mathcal{F}_{1} \rightarrow 0$ which may be viewed as deformations over the parameter space $\mathbb{C}[\epsilon] / \epsilon^{2}$ (and similarly for $\mathcal{F}_{2}$ ).

The conditions $\operatorname{Supp}\left(\mathcal{F}_{1} / \mathcal{F}_{2}\right)=x \in C$ and length $\left(\mathcal{F}_{1} / \mathcal{F}_{2}\right)=i$ imply that $\mathcal{F}_{1}(-i C) \subset \mathcal{F}_{2}$. Moreover, the following diagram

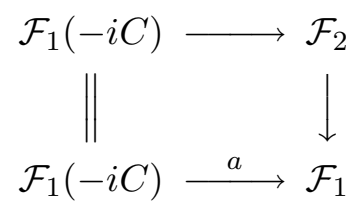


commutes. Here the map $a$ is the multiplication by $i$-th power of the local equation for $C$.

The diagram (5) should be preserved under infinitesimal deformation of the point $\left(\mathcal{F}_{1}, \mathcal{F}_{2}\right)$ within $P_{C}[-i]$. This means that:

(a) There exists a commutative diagram:

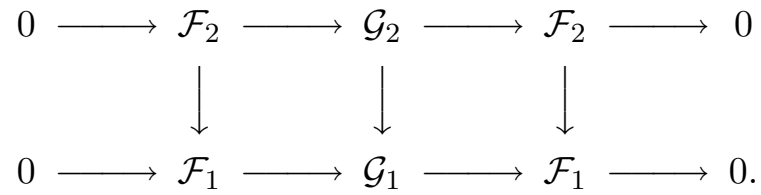

It is a standard fact of homological algebra that this is equivalent to requiring that the images of $v$ and $w$ under the natural maps $\operatorname{Ext}_{S}^{1}\left(\mathcal{F}_{1}, \mathcal{F}_{1}\right) \rightarrow \operatorname{Ext}_{S}^{1}\left(\mathcal{F}_{2}, \mathcal{F}_{1}\right)$ and $\operatorname{Ext}_{S}^{1}\left(\mathcal{F}_{2}, \mathcal{F}_{2}\right) \rightarrow \operatorname{Ext}_{s}^{1}\left(\mathcal{F}_{2}, \mathcal{F}_{1}\right)$ coincide.

(b) There exists a similar diagram of extensions corresponding to the embedding $\mathcal{F}_{1}(-i C) \subset \mathcal{F}_{2}$. This is can be expressed in terms of Ext groups in a similar way.

(c) The diagram of middile terms

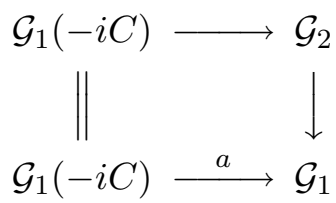

commutes. This condition can be expressed as vanishing of some homomorphism from the quotient copy of $\mathcal{F}_{1}(-i C)$ to the subsheaf copy of $\mathcal{F}_{1}$ (i.e., the embedding of sheaves $a$ should not be deformed). We will not write this down explicitly as we need this condition only in a special case (see below).

Step 3. We will prove that if $(v, w) \in W$ (where $W$ is the tangent space to the intersection $\left.p_{1}^{-1}\left(L^{\mu, s}\right) \cap P_{-i}^{C}\right)$, then $w \in T_{\mathcal{F}_{2}}\left(L^{\lambda, s}\right)$, and that $w=0$ implies $v=0$. The condition $w \in T_{\mathcal{F}_{2}}\left(L^{\lambda, s}\right)$ will follow from (a) above while the implication $(w=0) \Rightarrow(v=0)$ is a consequence of (c).

To use (a), consider the diagram:

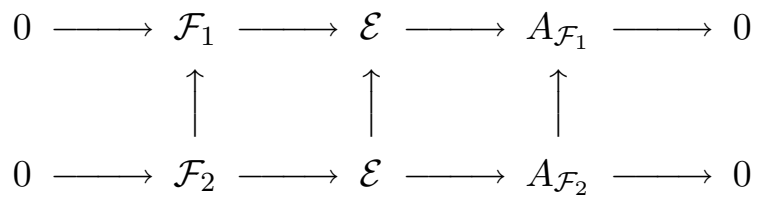


and the induced commutative digram of Ext-groups:

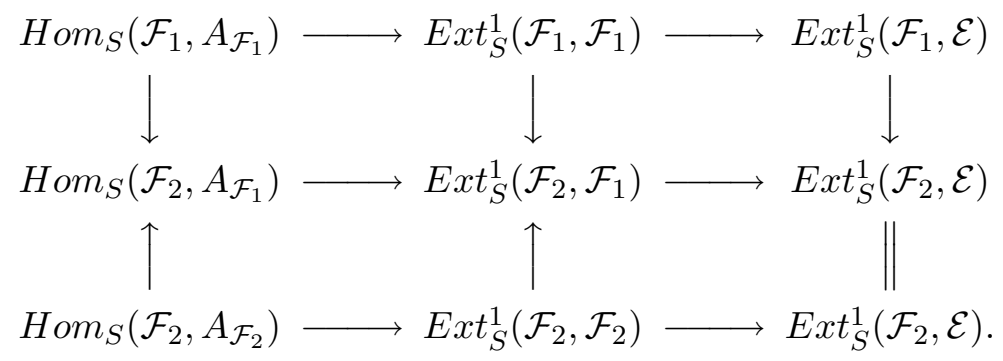

Here the lower two rows are obtained by applying $\operatorname{Hom}_{S}\left(\mathcal{F}_{2}, \cdot\right)$ to (6) and the upper row comes from applying $\operatorname{Hom}_{S}\left(\mathcal{F}_{2}, \cdot\right)$ to the upper row of $(6)$. Note that by stability $\operatorname{Hom}_{S}(\mathcal{E}, \mathcal{E})=\operatorname{Hom}_{S}\left(\mathcal{F}_{i}, \mathcal{F}_{i}\right)=$ $\operatorname{Hom}_{S}\left(\mathcal{F}_{i}, \mathcal{E}\right)=\mathbb{C}$ and hence the first arrow in each row is injective. Simple diagram chasing shows that if

$$
v \in \bigoplus_{x_{j} \in \operatorname{Supp}\left(A_{\mathcal{F}_{1}}\right)} \operatorname{Hom}_{S}\left(\mathcal{F}_{1} / \mathcal{E}\left(-\mu_{j} C\right),\left(A_{\mathcal{F}_{1}}\right)_{x_{j}}\right) \subset \operatorname{Hom}_{S}\left(\mathcal{F}_{1}, A_{\mathcal{F}_{1}}\right)
$$

then by the condition (b) we have

$$
w \in \bigoplus_{x_{j} \in \operatorname{Supp}\left(A_{\mathcal{F}_{2}}\right)} \operatorname{Hom}_{S}\left(\mathcal{F}_{2} / \mathcal{E}\left(-\lambda_{j} C\right),\left(A_{\mathcal{F}_{2}}\right)_{x_{j}}\right) \subset \operatorname{Hom}_{S}\left(\mathcal{F}_{2}, A_{\mathcal{F}_{2}}\right) .
$$

To prove injectivity, recall that for

$$
v \in \operatorname{Hom}_{S}\left(\mathcal{F}_{1}, A_{\mathcal{F}_{1}}\right) \subset \operatorname{Ext} t^{1}\left(\mathcal{F}_{1}, \mathcal{F}_{1}\right)
$$

the extension

$$
0 \rightarrow \mathcal{F}_{1} \rightarrow \mathcal{G}_{1} \rightarrow \mathcal{F}_{1} \rightarrow 0
$$

can be recovered as a kernel of the map of extensions

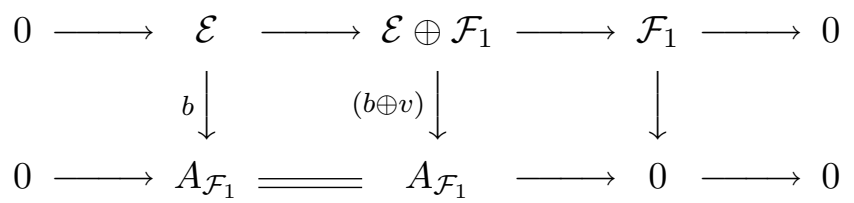

where $b$ is the cokernel of the natural embedding $\mathcal{F}_{1} \subset \mathcal{E}$. Similar statement is true for $w$ and the corresponding extension $0 \rightarrow \mathcal{F}_{2} \rightarrow$ 
$\mathcal{G}_{2} \rightarrow \mathcal{F}_{2} \rightarrow 0$. One shows that in our case the condition (c) translates into commutativity of the diagram

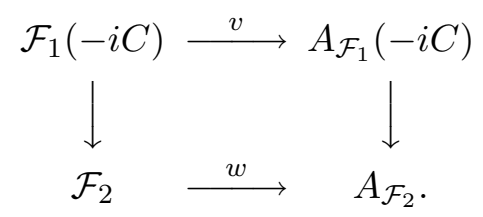

Since both vertical arrows in this diagram are injective the condition $w=0$ implies $v=0$.

This ends the proof of the lemma and hence the proof of Theorem 4.6. q.e.d.

\section{Computation of the intersection number}

The main result of this section is a computation of the intersection number of the fundamental classes of cycles $\operatorname{Quot}_{C}^{n}(\mathcal{E})$ and $\operatorname{Quot}_{C^{\prime}}^{n}(s)$ in $M^{G}(r, n+s)$. In the case of Hilbert schemes this intersection is transversal and the intersection number can be found by a simple set-theoretic argument (recall that we assume that $C$ and $C^{\prime}$ are transversal). However, for higher ranks the set-theoretic intersection is not transversal any more and to compute the intersection index we have to apply the excess intersection formula (cf. [11]).

By definition $\operatorname{Quot}_{C}^{n}(\mathcal{E}) \subset M^{G}(r, n+s)$ parametrizes all short exact sequences $\left.0 \rightarrow \mathcal{E}_{1} \rightarrow \mathcal{E}\right|_{C} \rightarrow A \rightarrow 0$ of sheaves on $C$, where $A$ is a length $n$ Artin sheaf. Any such sequence defines a sheaf $\mathcal{F} \in M^{G}(r, n+s)$ on $S$, namely the kernel of the composition $\left.\mathcal{E} \rightarrow \mathcal{E}\right|_{C} \rightarrow A$. Note that $\operatorname{Quot}_{C}^{n}(\mathcal{E})$ is smooth since the tangent space to it at a point represented by $\left.0 \rightarrow \mathcal{E}_{1} \rightarrow \mathcal{E}\right|_{C} \rightarrow A \rightarrow 0$ is $\operatorname{Hom}_{\mathcal{O}_{C}}\left(\mathcal{E}_{1}, A\right)$ which has a constant dimension $r n$ since $\mathcal{E}_{1}$ is a rank $r$ vector bundle on $C$, and $A$ is an Artin sheaf on $C$.

Similarly, $\operatorname{Quot}_{C^{\prime}}^{n}(s) \subset M^{G}(r, n+s)$ has open subset $\left(\operatorname{Quot}_{C^{\prime}}^{n}(s)\right)^{\circ}$ isomorphic to a fiber bundle over the subset $\left(M^{G}(r, s)\right)^{\circ}$ of locally free sheaves, with fiber $Q u o t_{\mathbb{C}^{\prime}}^{n}\left(\mathcal{E}^{\prime}\right)$ over $\mathcal{E}^{\prime} \in\left(M^{G}(r, s)\right)^{\circ}$. Hence $\left(\operatorname{Quot}_{C^{\prime}}^{n}(s)\right)^{\circ}$ is smooth.

Note that $\operatorname{Quot}_{C^{\prime}}^{n}(s) \backslash\left(\operatorname{Quot}_{C^{\prime}}^{n}(s)\right)^{\circ}$ does not intersect $Q u o t_{C}^{n}(\mathcal{E})$ since all points

$$
\mathcal{F} \in \operatorname{Quot}_{C}^{n}(\mathcal{E}) \subset M^{G}(r, n+s)
$$


satisfy length $\left(\mathcal{F}^{* *} / \mathcal{F}\right)=n$ and for sheaves in $\operatorname{Quot}_{C^{\prime}}^{n}(s) \backslash\left(\operatorname{Quot}_{C^{\prime}}^{n}(s)\right)^{\circ}$ this length is at least $(n+1)$.

Assume for the sake of simplicity that $S=\mathbb{P}^{2}$ and $C, C^{\prime}$ are two distinct lines intersecting at a point $x \in S$. The general case follows from our proof by a simple combinatorial argument.

Let $\mathcal{F} \in \operatorname{Quot}_{C^{\prime}}^{n}(s) \bigcap \operatorname{Quot}_{C}^{n}(\mathcal{E})$. Then $\mathcal{F}^{* *}=\mathcal{E}, \mathcal{F}$ is a kernel of $\left.\mathcal{E} \rightarrow \mathcal{E}\right|_{C} \rightarrow A$ and also a kernel of $\left.\mathcal{E} \rightarrow \mathcal{E}\right|_{C^{\prime}} \rightarrow A^{\prime}$. Hence $\mathcal{F}$ contains $\mathcal{E}(-C)+\mathcal{E}\left(-C^{\prime}\right)=\mathcal{E} \otimes \mathcal{J}_{x}$ as a subsheaf and $A \simeq A^{\prime} \simeq\left(\mathbb{C}_{x}\right)^{n}$, where $\mathcal{J}_{x}$ is the ideal sheaf of $x \in S$ and $\mathbb{C}_{x} \simeq \mathcal{O}_{S} / \mathcal{J}_{x}$. This means that every sheaf $F$ in our intersection can be obtained as a kernel of $\mathcal{E} \rightarrow \mathcal{E}_{x} \rightarrow A$ where $A$ is an $n$-dimensional quotient of an $r$-dimensional vector space $\mathcal{E}_{x}$. Hence $\operatorname{Quot}_{C^{\prime}}^{n}(s) \bigcap \operatorname{Quot}_{C}^{n}(\mathcal{E})$ is nothing but the Grassman variety $\operatorname{Gr}\left(\mathcal{E}_{x}, n\right)$ of all $n$-dimensional quotients of $\mathcal{E}_{x}$. In particular, $n \leq r$.

Since $\operatorname{Quot}_{C^{\prime}}^{n}(s)$ and $\operatorname{Quot}_{C}^{n}(\mathcal{E})$ are of complementary dimensions in the ambient $M^{G}(r, n+s)$ we see that for $r \geq 2$ their set-theoretic intersection has abnormally high dimension. However, it is true that $\operatorname{Quot}_{C^{\prime}}^{n}(s)$ and $\operatorname{Quot}_{C}^{n}(\mathcal{E})$ are smooth along the points of $G r\left(\mathcal{E}_{x}, n\right)$ and, moreover, the intersection is clean, i.e., for any $\mathcal{F} \in \operatorname{Gr}\left(\mathcal{E}_{x}, n\right)$

$$
T_{\mathcal{F}} \operatorname{Gr}\left(\mathcal{E}_{x}, n\right)=T_{\mathcal{F}} \operatorname{Quot}_{C^{\prime}}^{n}(s) \bigcap T_{\mathcal{F}} \operatorname{Quot}_{C}^{n}(\mathcal{E})
$$

(this can be checked using methods of the previous section).

Therefore, we can apply excess intersection formula (cf. [11, Example 6.1.7]): let $V$ be a rank $(r-n) n$ vector bundle on $\operatorname{Gr}\left(\mathcal{E}_{x}, n\right)$ arising form the following exact sequence:

$$
0 \rightarrow T_{G r(r, n)} \rightarrow T_{Q u o t_{C}^{n}(\mathcal{E})} \oplus T_{Q u o t_{C^{\prime}}^{n}(s)} \rightarrow T_{M^{G}(r, n+s)} \rightarrow V \rightarrow 0
$$

Then the intersection number $\left[\operatorname{Quot}_{C}^{n}(\mathcal{E})\right] \cdot\left[\operatorname{Quot}_{C^{\prime}}^{n}(s)\right]$ in $M^{G}(r, n+s)$ is equal to the top Chern class $c_{(r-n) n}(V)$.

To compute the Chern class of the previous section we need to consider certain sheaves on $M^{G}(r, n+s) \times S$.

Notation. For any pair of sheaves $\mathcal{G}_{1}, \mathcal{G}_{2}$ on $M^{G}(r, n+s) \times S$ let $\mathcal{E} x t_{p_{1}}^{i}\left(\mathcal{G}_{1}, \mathcal{G}_{2}\right)$ be the relative Ext-sheaf on $M^{G}(r, n+s)$ with respect to the first projection $p_{1}: M^{G}(r, n+s) \times S \rightarrow M^{G}(r, n+s)$.

We will only deal with the cases when for all $x \in M^{G}(r, n+s)$ the global Ext group $\operatorname{Ext}^{i}\left(p_{1}^{-1}(x) ; \mathcal{G}_{1}, \mathcal{G}_{2}\right)$ on the fiber $p_{1}^{-1}(x)$ is of constant dimension. Hence by [19] $\mathcal{E} x t_{p_{1}}^{i}\left(\mathcal{G}_{1}, \mathcal{G}_{2}\right)$ is a vector bundle on 
$M^{G}(r, n+s)$ which has the global Ext-group above as the fiber over point $x$. Similar remarks apply to any closed subspace of $M^{G}(r, n+s)$ (abusing notation we will denote a sheaf on $M^{G}(r, n+s)$ and its restriction to a closed subspace by the same letter).

Since $\operatorname{gcd}\left(r, c_{1}(H) \cdot c_{1}(L)\right)=1$, there exists a universal sheaf $\mathcal{G}$ on $M^{G}(r, n+s) \times S$ (cf. [17]). It is known (cf. [22]) that the tangent bundle $T_{M^{G}(r, n+s)}$ is isomorphic to the kernel of the surjective map of the vector bundles

$$
\mathcal{E} x t_{p_{1}}^{1}(\mathcal{G}, \mathcal{G}) \rightarrow H^{1}\left(S, \mathcal{O}_{S}\right) \otimes \mathcal{O}_{M^{G}(r, n+s)} \rightarrow 0 .
$$

In particular, the tangent bundle to $M^{G}(r, n+s)$ has the same Chern classes as $\mathcal{E} x t_{p_{1}}^{1}(\mathcal{G}, \mathcal{G})$.

Lemma 6.1. Let $Q$ be the universal quotient bundle on the Grassman variety $\operatorname{Gr}\left(\mathcal{E}_{x}, n\right)$. The the full Chern class of $\left.\mathcal{E} x t_{p_{1}}^{1}(\mathcal{G}, \mathcal{G})\right|_{G r\left(\mathcal{E}_{x}, n\right)}$ is equal to $\left(c(Q) c\left(Q^{*}\right)\right)^{r}$, where $c(Q)$ (resp. $\left.c\left(Q^{*}\right)\right)$ is the full Chern class of $Q$ (resp. its dual).

Proof. Note that $\left.\mathcal{G}\right|_{\operatorname{Gr}\left(\mathcal{E}_{x}, n\right) \times S}$ can be included in the short exact sequence of bundles on $\operatorname{Gr}\left(\mathcal{E}_{x}, n\right) \times S$ :

$$
0 \rightarrow \mathcal{G} \rightarrow p_{2}^{*} \mathcal{E} \rightarrow \mathcal{A} \rightarrow 0
$$

where $p_{1}, p_{2}$ are the projections, $\mathcal{A}=p_{1}^{*} Q \otimes p_{2}^{*} \mathbb{C}_{x}$ and $Q$ is the universal quotient bundle on the Grassmanian.

Applying $R \mathcal{H}$ om $_{p_{1}}(\cdot, \mathcal{G})$ to $(8)$ one obtains a long exact sequence of sheaves on the Grassmanian:

$$
\begin{aligned}
0 \rightarrow \mathcal{H o m}_{p_{1}}(\mathcal{G}, \mathcal{G}) & \rightarrow \mathcal{E} x t_{p_{1}}^{1}(\mathcal{A}, \mathcal{G}) \rightarrow \mathcal{E} x t_{p_{1}}^{1}\left(p_{2}^{*} \mathcal{E}, \mathcal{G}\right) \rightarrow \\
& \rightarrow \mathcal{E} x t_{p_{1}}^{1}(\mathcal{G}, \mathcal{G}) \rightarrow \mathcal{E} x t_{p_{1}}^{2}(\mathcal{A}, \mathcal{G}) \rightarrow 0
\end{aligned}
$$

Indeed, $\mathcal{H o m}_{p_{1}}\left(p_{2}^{*} \mathcal{E}, \mathcal{G}\right)$ is zero since its fiber over $\mathcal{F} \in \operatorname{Gr}\left(\mathcal{E}_{x}, n\right)$ is $\operatorname{Hom}_{\mathcal{O}_{S}}(\mathcal{E}, \mathcal{F})$ which is zero $\left(\mathcal{E}\right.$ and $\mathcal{F}$ are stable and $\left.c_{2}(\mathcal{F})>c_{2}(\mathcal{E})\right)$. We can put zero on the end because, due to the Technical Condition of Section 1 , either both $\mathcal{E} x t_{p_{1}}^{2}\left(p_{2}^{*} \mathcal{E}, \mathcal{G}\right)$ and $\mathcal{E} x t_{p_{1}}^{2}(\mathcal{G}, \mathcal{G})$ are zero (when $c_{1}(K) \cdot c_{1}(H)<0$ ) or the map between them is an isomorphism (when $K \simeq \mathcal{O})$. Moreover, $\mathcal{H o m}_{p_{1}}(\mathcal{G}, \mathcal{G})$ is a trivial line bundle, since its fiber $\mathcal{F}$ is $\operatorname{Hom}_{\mathcal{O}_{S}}(\mathcal{F}, \mathcal{F})$ and stable sheaves have only scalar automorphisms.

Thus, the full Chern class of $T_{M^{G}(r, n+s)}$ is equal to:

$$
c\left(T_{M^{G}(r, n+s)}\right)=c\left(\mathcal{E} x t_{p_{1}}^{1}(\mathcal{G}, \mathcal{G})\right)=\frac{c\left(\mathcal{E} x t_{p_{1}}^{2}(\mathcal{A}, \mathcal{G})\right) c\left(\mathcal{E} x t_{p_{1}}^{1}\left(p_{2}^{*} \mathcal{E}, \mathcal{G}\right)\right)}{c\left(\mathcal{E} x t_{p_{1}}^{1}(\mathcal{A}, \mathcal{G})\right)} .
$$


Now we proceed to compute the three terms in the formula above.

To compute $c\left(\mathcal{E} x t_{p_{1}}^{1}\left(p_{2}^{*} \mathcal{E}, \mathcal{G}\right)\right)$ we apply $R \mathcal{H} \operatorname{Hom}_{p_{1}}\left(p_{2}^{*} \mathcal{E}, \cdot\right)$ to $(8)$ and get

$$
\begin{aligned}
0 \rightarrow \mathcal{H o m}_{p_{1}}\left(p_{2}^{*} \mathcal{E}, p_{2}^{*} \mathcal{E}\right) & \rightarrow \mathcal{H o m}_{p_{1}}\left(p_{2}^{*} \mathcal{E}, \mathcal{A}\right) \rightarrow \\
& \rightarrow \mathcal{E} x t_{p_{1}}^{1}\left(p_{2}^{*} \mathcal{E}, \mathcal{G}\right) \rightarrow \mathcal{E} x t_{p_{1}}^{1}\left(p_{2}^{*} \mathcal{E}, p_{2}^{*} \mathcal{E}\right) \rightarrow 0
\end{aligned}
$$

Here, the sheaf $\mathcal{H o m}_{p_{1}}\left(p_{2}^{*} \mathcal{E}, \mathcal{G}\right)$ vanishes as before and $\mathcal{E} x t^{1}\left(p_{2}^{*} \mathcal{E}, \mathcal{A}\right)$ is zero since its fiber over a point $\mathcal{F}$ is $\operatorname{Ext}_{\mathcal{O}_{S}}^{1}\left(\mathcal{E},\left(\mathbb{C}_{x}\right)^{n}\right)=H^{1}\left(S, \mathcal{E}^{*} \otimes\right.$ $\left.\left(\mathbb{C}_{x}\right)^{n}\right)=0$. The bundles $\mathcal{H o m}_{p_{1}}\left(p_{2}^{*} \mathcal{E}, p_{2}^{*} \mathcal{E}\right)$ and $\mathcal{E} x t_{p_{1}}^{1}\left(p_{2}^{*} \mathcal{E}, p_{2}^{*} \mathcal{E}\right)$ are trivial on $\operatorname{Gr}\left(\mathcal{E}_{x}, n\right)$ hence

$$
c\left(\mathcal{E} x t_{p_{1}}^{1}\left(p_{2}^{*} \mathcal{E}, \mathcal{G}\right)\right)=c\left(\mathcal{H o m}_{p_{1}}\left(p_{2}^{*} \mathcal{E}, \mathcal{A}\right)\right)=c\left(Q \otimes \mathbb{C}^{r}\right)=(c(Q))^{r} .
$$

To compute $c\left(\mathcal{E} x t_{p_{1}}^{1}(\mathcal{A}, \mathcal{G})\right)$ we apply $R \mathcal{H} \operatorname{Hom}_{p_{1}}(\mathcal{A}, \cdot)$ to $(8)$ and get

$$
\begin{aligned}
0 & \rightarrow \mathcal{H o m}_{p_{1}}(\mathcal{A}, \mathcal{A}) \rightarrow \mathcal{E} x t_{p_{1}}^{1}(\mathcal{A}, \mathcal{G}) \rightarrow 0 \rightarrow \mathcal{E} x t_{p_{1}}^{1}(\mathcal{A}, \mathcal{A}) \rightarrow \\
& \rightarrow \mathcal{E} x t_{p_{1}}^{2}(\mathcal{A}, \mathcal{G}) \rightarrow \mathcal{E} x t_{p_{1}}^{2}\left(\mathcal{A}, p_{2}^{*} \mathcal{E}\right) \rightarrow \mathcal{E} x t_{p_{1}}^{2}(\mathcal{A}, \mathcal{A}) \rightarrow 0
\end{aligned}
$$

where $\mathcal{E} x t_{p_{1}}^{1}\left(\mathcal{A}, p_{2}^{*} \mathcal{E}\right)$ vanishes since it is Serre dual to $\mathcal{E} x t_{p_{1}}^{1}\left(p_{2}^{*} \mathcal{E}, \mathcal{A} \otimes\right.$ $p_{2}^{*} K_{S}$ ) (cf. [19]), and the fibers of the latter sheaf compute first cohomology of an Artin sheaf. Since $\mathcal{H o m}_{p_{1}}(\mathcal{A}, \mathcal{A}) \simeq Q \otimes Q^{*}$, we conclude that $c\left(\mathcal{E} x t_{p_{1}}^{1}(\mathcal{A}, \mathcal{G})\right)=c\left(Q \otimes Q^{*}\right)$.

To compute $c\left(\mathcal{E} x t_{p_{1}}^{2}(\mathcal{A}, \mathcal{G})\right)$ we use the exact sequence from the computation of $c\left(\mathcal{E} x t_{p_{1}}^{1}(\mathcal{A}, \mathcal{G})\right)$. From $\operatorname{Ext}_{\mathcal{O}_{S}}^{2}\left(\mathbb{C}_{x}, \mathbb{C}_{x}\right)=\mathbb{C}$ and $\operatorname{Ext}_{\mathcal{O}_{S}}^{1}\left(\mathbb{C}_{x}, \mathbb{C}_{x}\right)$ $=\mathbb{C}^{2}$, we deduce

$$
\operatorname{Ext}_{p_{1}}^{2}(\mathcal{A}, \mathcal{A}) \simeq Q \otimes Q^{*}, \quad \operatorname{Ext}_{p_{1}}^{1}(\mathcal{A}, \mathcal{A})=\left(Q \otimes Q^{*}\right)^{\oplus 2} .
$$

therefore, it only remains to compute $\mathcal{E} x t_{p_{1}}^{2}\left(\mathcal{A}, p_{2}^{*} \mathcal{E}\right)$. To that end, we again apply a relative version of Serre's duality (loc. cit.) which gives

$$
\mathcal{E} x t_{p_{1}}^{2}\left(\mathcal{A}, p_{2}^{*} \mathcal{E}\right) \simeq\left(Q^{*}\right)^{\oplus r} .
$$

and hence $c\left(\mathcal{E} x t_{p_{1}}^{2}(\mathcal{A}, \mathcal{G})\right)=c\left(Q \otimes Q^{*}\right)\left(c\left(Q^{*}\right)\right)^{r}$.

Summing up the results of our computation, we obtain: 


$$
c\left(T_{M^{G}(r, n+s)}\right)=\frac{c\left(Q \otimes Q^{*}\right)\left(c\left(Q^{*}\right)\right)^{r} \cdot(c(Q))^{r}}{c\left(Q \otimes Q^{*}\right)}=\left(c(Q) c\left(Q^{*}\right)\right)^{r} .
$$

q.e.d.

A similar approach can be used with $T_{Q u o t}^{n}(\mathcal{E})$ and $T_{Q u o t^{\prime}}^{n}(s)$ (one has to consider sheaves on $\operatorname{Gr}\left(\mathcal{E}_{x}, n\right) \times C$ and $\left.\operatorname{Gr}\left(\mathcal{E}_{x}, n\right) \times C^{\prime}\right)$. This leads to:

Lemma 6.2. The following equalities for the full Chern classes of sheaves on $\operatorname{Gr}\left(\mathcal{E}_{x}, n\right)$ hold:

$$
c\left(T_{Q_{\text {uot }}^{n}(\mathcal{E})}\right)=c\left(T_{Q_{C^{\prime}}}^{n}(s)\right)=(c(Q))^{r},
$$

where $c(Q)$ is the full Chern class of the universal quotient bundle.

Let $S$ be the universal subbundle on $\operatorname{Gr}\left(\mathcal{E}_{x}, n\right)$. Then $T_{G r\left(\mathcal{E}_{x}, n\right)} \simeq$ $S^{*} \otimes Q$. Now using the exact sequence $(7)$ we obtain

$$
\begin{aligned}
c(V) & =\frac{c\left(T_{M^{G}(r, n+s)}\right) c\left(T_{G r\left(\mathcal{E}_{x}, n\right)}\right)}{c\left(T_{Q u o t_{C}^{n}(\mathcal{E})}\right) c\left(T_{Q_{\text {uot }_{C^{\prime}}^{n}}(s)}\right)}=\frac{\left(c(Q) c\left(Q^{*}\right)\right)^{r}\left(c\left(S^{*} \otimes Q\right)\right)}{(c(Q))^{r}(c(Q))^{r}} \\
& =\frac{\left(c\left(Q^{*}\right)\right)^{r}\left(c\left(S^{*} \otimes Q\right)\right)}{(c(Q))^{r}}=\frac{\left(c\left(Q^{*}\right)\right)^{r}}{c\left(Q \otimes Q^{*}\right)} \\
& =c\left(Q^{*} \otimes S\right) .
\end{aligned}
$$

The equality in the second line is obtained by applying $(\ldots) \otimes Q$ to the dual of the tautological exact sequence $0 \rightarrow S \rightarrow \mathcal{O}^{r} \rightarrow Q \rightarrow 0$. The last equality is obtained by applying $(\ldots) \otimes Q^{*}$ to the tautological sequence itself.

Note that $S^{*} \otimes Q$ is the cotangent bundle to the Grassmanian. Its top Chern class (which can be computed from the topological Euler characteristic of $\left.\operatorname{Gr}\left(\mathcal{E}_{x}, n\right)\right)$ is equal to $(-1)^{(r-1) n}\left(\begin{array}{l}r \\ n\end{array}\right)$. Recall that this number computes the intersection index of the cycles $\left[\operatorname{Quot}_{C}^{n}(\mathcal{E})\right]$ and $\left[\operatorname{Quot}_{C^{\prime}}^{n}(s)\right]$ in the case when $C$ and $C^{\prime}$ are a pair of distinct lines on $\mathbb{P}^{2}$. In the general case, the curves $C$ and $C^{\prime}$ will intersect in $\left\langle C, C^{\prime}\right\rangle$ points. Then we have the

\section{Corollary 6.3.}

$$
\sum_{n=1}^{\infty} z^{2 n}\left\langle\operatorname{Quot}_{C}^{n}(\mathcal{E}), \text { Quot }_{C^{\prime}}^{n}(s)\right\rangle=\left(1+(-1)^{r-1} z^{2}\right)^{r\left\langle C, C^{\prime}\right\rangle}
$$


Proof. Denote $\left\langle C, C^{\prime}\right\rangle$ by $q$ and $(-1)^{(r-1) n}\left(\begin{array}{l}r \\ n\end{array}\right)$ by $a_{n}$. It follows from the definitions and the discussion in the beginning of this chapter, that the set-theoretic intersection $\operatorname{Quot}_{C}^{n}(\mathcal{E}) \cap Q u o t_{C^{\prime}}^{n}(s)$ corresponds to sheaves $\mathcal{F}$ which fit into a short exact sequence $0 \rightarrow \mathcal{F} \rightarrow \mathcal{E} \rightarrow \mathcal{A}$, such that $\mathcal{A}$ is actually a quotient of $\bigoplus_{x \in C \cap C^{\prime}} \mathcal{E}_{x}$. The variety of such sheaves has several irreducible components according to the multiplicity of $\mathcal{A}$ at the points on $C \cap C^{\prime}$. Thus, each component is labeled by a subdivision of $n$ into a sum $n_{1}+\ldots+n_{q}$ of $q$ ordered nonnegative integers. Such a component is a product of the Grassmanians $\prod_{x_{i} \in C \cap C^{\prime}} \operatorname{Gr}\left(\mathcal{E}_{x}, n_{i}\right)$, and it has the excess intersection bundle as before. It is easy to show that the top Chern class of this bundle will be equal to the product $a_{n_{1}} \cdot \ldots \cdot a_{n_{q}}$ (since all data will split into direct product over the individual Grassmanians). Thus, to get the intersection number $\left\langle Q u o t_{C}^{n}(\mathcal{E}), \operatorname{Quot}_{C^{\prime}}^{n}(s)\right\rangle$ we need to sum up the products $a_{n_{1}} \cdot \ldots \cdot a_{n_{q}}$ over all subdivisions $n=n_{1}+\ldots+n_{q}$. Since $a_{k}=(-1)^{(r-1) k}\left(\begin{array}{l}r \\ k\end{array}\right)$, a standard combinatorial argument and the binomial formula show that $\sum_{n=1}^{\infty} z^{2 n}\left\langle\operatorname{Quot}_{C}^{n}(\mathcal{E}), \operatorname{Quot}_{C^{\prime}}^{n}(s)\right\rangle=\left(1+(-1)^{r-1} z^{2}\right)^{r\left\langle C, C^{\prime}\right\rangle}$. q.e.d.

\section{A. Appendix: The punctual quot scheme}

In this appendix we outline the proof of Theorem 2.2 which claims that the punctual Quot scheme $\operatorname{Quot}(r, n)$ is irreducible of dimension $r n-1$. The full details can be found in [2].

Since the main object of interest in this appendix is local in its nature, we will assume that the surface $S$ is $\mathbb{C}^{2}$. Recall that the closed points of $Q u o t(r, n)$ are in bijective correspondence with all length $n$ Artin quotients $\mathcal{O}_{\mathbb{C}^{2}}^{r} \rightarrow \mathcal{A}$ which are supported at the origin. This allows us to give a description of $\operatorname{Quot}(r, n)$ in terms of linear algebra, generalizing Nakajima's construction for Hilbert schemes, cf. [27].

Let $\mathcal{A}$ be such a quotient and choose an isomorphism of $H^{0}\left(\mathbb{C}^{2}, \mathcal{A}\right)$ with a standard $n$-dimensional vector space $V$. Then multiplication by the two coordinate functions on $\mathbb{C}^{2}$ gives two commuting operators $B_{1}$ and $B_{2}$ on $V$. It is easy to see that $\mathcal{A}$ is supporeted at $0 \in \mathbb{C}^{2}$ if and only if $B_{1}$ and $B_{2}$ are nilpotent. Moreover, the $r$ natural constant sections of $\mathcal{O}_{\mathbb{C}^{2}}^{r}$ map to certain vectors $v_{1}, \ldots, v_{r}$ in $V$ which satisfy the following

Definition. Let $V$ be a vector space with two operators $B_{1}, B_{2}$. A 
set of vectors $v_{1}, \ldots, v_{r}$ is called cyclic if there are no proper subspaces $W \subset V$, such that

(a) $v_{i} \in W, i=1, \ldots, r$,

(b) $B_{1}(W) \subset W, B_{2}(W) \subset W$.

Let $\mathcal{N}_{2}$ be the space of pairs of commuting nilpotent operators on $V$, a closed affine subvariety of $\operatorname{End}(V) \oplus \operatorname{End}(V)$.

Consider also a subspace $U_{r}$ of $\mathcal{N}_{2} \times V^{\oplus r}$ formed by all $\left(B_{1}, B_{2}, v_{1}\right.$, $\left.\ldots, v_{r}\right)$, such that $v_{1}, \ldots, v_{r}$ is a cyclic set of vectors. It is easy to see that $U_{r}$ is an open subset in $\mathcal{N}_{2} \times V^{\oplus r}$ (it is given by a condition saying that some system of vectors in $V$ has maximal rank). The group $G L(V)$ acts on $U_{r}$ by conjugating the nilpotent operators and acting on vectors diagonally.

Lemma A.1. $G L(V)$ acts freely on $U_{r}$.

Proof. Suppose $g \in G L(V)$ stabilizes $\left(B_{1}, B_{2}, v_{1}, \ldots, v_{r}\right) \in U_{r}$. Then $\operatorname{Ker}(1-g)$ contains $v_{1}, \ldots, v_{r}$. Since it is also preserved by $B_{1}, B_{2}$, we have $\operatorname{Ker}(1-g)=V$ and therefore $g=1$. q.e.d.

The following lemma gives an explicit construction of the punctual Quot scheme:

Lemma A.2. There exists a morphism $\pi: U_{r} \rightarrow$ Quot $(r, n)$ such that:

(i) $\pi$ is surjective;

(ii) The fibers of $\pi$ are precisely the orbits of $G L(V)$ action on $U_{r}$.

Proof. Clear from the discussion preceding the definition of a cyclic set of vectors. q.e.d.

Our strategy in proving Theorem 2.2 is as follows. First we note that we have

Theorem A.3. $U_{1}$ is irreducible of dimension $n^{2}+n-1$.

Proof. Follows from the previous Lemma and the irreduciblity and dimension of $\operatorname{Hilb}^{n}=\operatorname{Quot}(1, n)$ proved in [5], [18]. q.e.d.

Once we manage to show that $U_{1}$ is dense in $\mathcal{N}_{2}$, that will imply that $\mathcal{N}_{2}$ is irreducible of dimension $n^{2}-1$. Since $U_{r}$ is open in $\mathcal{N}_{2} \times V^{\oplus r}$, by 
Lemma A.2 we will be able to prove the corresponding statements for Quot $(r, n)$.

Lemma A.4 (cf. [2]). Let $B_{1}, B_{2}$ be two commuting nilpotent operators on a vector space $V$. There exists a third nilpotent operator $B_{2}^{\prime}$ and a vector $w \in V$ such that:

(i) $B_{2}^{\prime}$ commutes with $B_{1}$;

(ii) Any linear combination $\alpha B_{2}+\beta B_{2}^{\prime}$ is nilpotent;

(iii) $w$ is a cyclic vector for the pair of operators $\left(B_{1}, B_{2}^{\prime}\right)$.

Proof. See Lemma 3 in [2]. $\quad$ q.e.d.

Theorem A.5. The subset $U_{1}$ defined above is dense in $\mathcal{N}_{2} \times V$.

Proof. Let $\left(B_{1}, B_{2}, v\right)$ be any point in $\mathcal{N}_{2} \times V$. Consider the triple $\left(B_{1}, B_{2}^{\prime}, w\right) \in U$ provided by the above lemma. Connect the two triples $\left(B_{1}, B_{2}, v\right)$ and $\left(B_{1}, B_{2}^{\prime}, w\right)$ by an affine line $L$ inside the vector space $g l(V) \oplus g l(V) \oplus V$. Due to the choice of $B_{2}^{\prime}$ the whole line $L$ belongs to the closed subspace $\mathcal{N}_{2} \times V \subset g l(V) \oplus g l(V) \oplus V$. Since $U \cap L$ is open in $L$ and non-empty, $\left(B_{1}, B_{2}, v\right)$ belongs to the closure of $U$. q.e.d.

Corollary A.6. The punctual Quot scheme Quot $(r, n)$ is irreducible of dimension $r n-1$.

Proof. By the previous theorem $\mathcal{N}_{2}$ is irreducible of dimension $n^{2}-1$. Since $U_{r}$ is dense in $\mathcal{N}_{2} \times V^{\oplus r}$, it is irreducible of dimension $n^{2}-1+r n$. Finally, by Lemma A.2 the variety $U_{r}$ is a principal $G L(V)$ bundle over $\operatorname{Quot}(r, n)$ therefore $\operatorname{Quot}(r, n)$ is irreducible of dimension $r n-1$. q.e.d.

\section{References}

[1] I. V. Artamkin, Deformation of torsion-free sheaves on an algebraic surface, (Russian) Izv. Akad. Nauk SSSR Ser. Mat. 54 (1990), no. 3, 435-468; translation in Math. USSR-Izv. 36 (1991), no. 3, 449-485.

[2] V. Baranovsky, The variety of pairs of commuting nilpotent matrices is irreducible, Transformation Groups 6 (2001) 3-8.

[3] A. A. Beilinson, J. Bernstein \& P. Deligne, Faisceaux pervers, Analysis and topology on singular spaces. I, (Luminy, 1981), 5-171, Astérisque, Vol. 100, Soc. Math. France, Paris, 1982. 
[4] W. Borho \& R. MacPherson, Partial resolutions of nilpotent varieties, Analysis and topology on singular spaces. II, III, (Luminy, 1981), 23-74, Astérisque, Vol. 101-102, Soc. Math. France, Paris, 1983.

[5] J. Briançon, Description de Hilb $^{n} C\{x, y\}$, Invent. Math. 41 (1977) 45-89.

[6] J. Burillo, The Poincaré-Hodge polynomial of a symmetric product of compact Kähler manifolds, (Spanish) Collect. Math. 41 (1990) 59-69.

[7] Chriss, N.; Ginzburg, V.: Representation theory and complex geometry. Birkhäuser Boston, Inc., Boston, MA, 1997.

[8] S. K. Donaldson \& P. B. Kronheimer, The geometry of four-manifolds, Oxford Math. Monographs, Oxford Science Publ. The Clarendon Press, Oxford Univ. Press, New York, 1990.

[9] G. Ellingsrud \& M. Lehn, On the irreducibility of the punctual Quotient Scheme of a surface, Ark. Mat. 37 (1999) 245-254.

[10] J. Fogarty, Algebraic families on an algebraic surface, Amer. J. Math. 90 (1968) 511-521.

[11] W. Fulton, Intersection theory, Ergebnisse der Mathematik und ihrer Grenzgebiete (3), 2. Springer, Berlin-New York, 1984.

[12] L. Göttsche, The Betti numbers of the Hilbert scheme of points on a smooth projective surface, Math. Ann. 286 (1990) 193-207.

[13] Theta functions and Hodge numbers of moduli spaces of sheaves on rational surfaces, Comm. Math. Phys. 206 (1999) 105-136.

[14] L. Göttsche, \& W. Soergel, Perverse sheaves and the cohomology of Hilbert schemes of smooth algebraic surfaces, Math. Ann. 296 (1993) 235-245.

[15] I. Grojnowski, Instantons and affine algebras. I. The Hilbert scheme and vertex operators, Math. Res. Lett. 3 (1996) 275-291.

[16] A. Grothendieck, Techniques de construction et théorémes d'existence en géométrie algébrique IV: Les schémas de Hilbert, Séminaire Bourbaki 221 (1960/61).

[17] D. Huybrechts, \& M. Lehn, The geometry of moduli spaces of sheaves, Aspects of Math., E31. Friedr. Vieweg \& Sohn, Braunschweig, 1997.

[18] A. Iarrobino, Punctual Hilbert schemes, Mem. Amer. Math. Soc. 10 (1977).

[19] S. Kleiman, Relative duality for quasicoherent sheaves, Compositio. Math. 41 (1980) 39-60.

[20] A. Kuznetsov, The Laumon's resolution of Drinfeld's compactification is small, Preprint alg-geom/9610019.

[21] J. Li, Algebraic geometric interpretation of Donaldson's polynomial invariant, J. Differential Geometry 37 (1993) 417-466. 
[22] M. Maruyama, Moduli of stable sheaves. I, II, J. Math. Kyoto Univ. 17 (1977) 91-126; 18 (1978) 557-614.

[23] I. G. Macdonald, The Poincaré polynomial of a symmetric product, Proc. Camb. Phil. Soc. 58 (1962) 563-568.

[24] _ Symmetric functions and Hall polynomials, Second ed. With contributions by A. Zelevinsky. Oxford Math. Monographs, Oxford Science Publications. The Clarendon Press, Oxford Univ. Press, New York, 1995.

[25] J. W. Morgan, Comparison of the Donaldson polynomials with their algebrogeometric analogues, Topology 32 (1993) 449-488.

[26] H. Nakajima, Instantons on ALE spaces, quiver varieties and Kac-Moody algebras, Duke Math. J. 76 (1994) 365-416.

[27] Lectures on Hilbert schemes of points on surfaces, University Lecture Ser., 18, Amer. Math. Soc., Providence, RI, 1999.

[28] C. Okonek, M. Schneider \& H. Spindler, Vector bundles on complex projective spaces, Progr. in Math. Vol. 3, Birkhäuser, Boston, MA, 1980.

[29] W.-P. Li, \& Z. Qin, On blowup formulae for the S-duality conjecture of Vafa and Witten. I, II, Preprints math.AG/9805054, math.AG/9805055.

[30] M. Saito, Introduction to mixed Hodge modules, Actes du Colloque de Théorie de Hodge (Luminy, 1987). Astérisque No. 179-180, 1989, 145-162.

[31] K. K. Uhlenbeck, Connections with $L^{p}$ bounds on curvature, Comm. Math. Phys. 83 (1982) 31-42.

[32] C. Vafa \& E. Witten, A strong coupling test of S-duality, Preprint hep-th/9408074.

[33] K. Yoshioka, The Betti numbers of the moduli space of stable sheaves of rank 2 on $\mathbf{P}^{2}$, J. Reine Angew. Math. 453 (1994) 193-220.

CALteCH 